\title{
Geothermobarometry of amphiboles in intermediate to basic rocks from the Almogholagh pluton in western Iran
}

\author{
Manuchehr Amiri, Ahmad Ahmadi Khalaji, Zahra TAhmasbi, Reza Zarei SAhamieh and Hassan Zamanian \\ Department of Geology, Faculty of Science, Lorestan University, Khoramabad, Iran
}

\begin{abstract}
In this study, some barometries and thermometries were used to calculate the crystallization temperature and pressure of amphiboles in intermediate to basic rocks from the Almogholagh pluton in western Iran. Intersection among geothermometers was introduced as a method to estimate crystallization temperature, and the results obtained were compared with those of other methods. Results showed that obtaining the average pressure from the intersections between geothermometers and geobarometers is a suitable approach to determine the crystallization pressure of the analyzed points of amphiboles, whereas the crystallization temperature can be appropriately determined from the intersection among geothermometers. In addition, the average temperature of the $\mathrm{HB}_{2} 94$ geothermometer is approximately equal to the average temperature in intersection among geothermometers. $\mathrm{HB}_{2} 94$ is apparently a suitable geothermometer to determine the crystallization temperature in intermediate to basic rocks. In this study, the average crystallization temperature and pressure using intersection methods were $714-719^{\circ} \mathrm{C}$ and 6.5-7.5 kbar, respectively. Adopting these methods to the Almogholagh pluton, intermediate to basic rocks intruded at a depth of $24-28 \mathrm{~km}$ of the crust and at $674-759{ }^{\circ} \mathrm{C}$.
\end{abstract}

Keywords: Almogholagh pluton, Amphibole, Geobarometry, Geothermobarometry, Geothermometry

\section{INTRODUCTION}

Amphiboles are chemically and structurally complex minerals. According to Leake (1978), with the approval of the International Mineralogical Association (IMA), the subcommittee on amphiboles has presented the classification of amphiboles (Leake et al., 1997, 2004). This classification is important for geothermobarometric studies. Some amphiboles, such as actinolite, are created through subsolidus and secondary processes (Leake, 1971; Helmy et al., 2004) and thus are not used in geothermobarometric studies. Actinolite is identified from other amphiboles on the basis of diagrams in the above-mentioned amphibole classification.

Amphibole composition varies depending on the bulk composition of magma, temperature, oxygen fugacity, and pressure during crystallization. Al content in amphibole is affected by the four aforementioned factors. Increasing temperature and pressure increase $\mathrm{Al}_{2} \mathrm{O}_{3}$ content in amphiboles, and their effects are considerably greater than those of the two other factors (Moody et al., 1983). In

doi:10.2465/jmps. 151228

M. Amiri, amiran1342@gmail.com Correspnding author the last two decades, Al geobarometry in amphiboles has been widely used to calculate the crystallization pressure of magma and to determine the emplacement depth of batholiths in the earth's crust (e.g., Ague and Brandon, 1992; Anderson and Smith, 1995). Geobarometers are generally divided into two groups: empirical and experimental. Empirical geobarometers are based on calcic amphiboles in calc-alkalic masses. This type of geobarometers includes that of Hammarstrom and Zen (1986) and Hollister et al. (1987), referred to as Formulas 1 (Ham in Table 6) and 2 (Hol) in the APPENDIXES. Experimental geobarometers, such as those developed by Johnson and Rutherford (1989) and Schmidt (1992), are shown as Formulas 3 (Joh) and 4 (Sch) in the APPENDIXES. The pressures in the Formula of Schmidt (1992) are similar to and thus confirm those achieved from the contact aureole of igneous masses (Ague, 1997). Using the data of Schmidt (1992), Anderson and Smith (1995) designed a geobarometer [shown as Formula 5 (And) in the APPENDIXES] that considers the effect of temperature.

Hornblende-plagioclase thermometry is the most commonly used method for calc-alkaline igneous rocks (Stein and Dietl, 2001; Ernst, 2002). Adopting an ideal structure for amphiboles and a non-ideal structure for pla- 
gioclases, Blundy and Holland (1990) developed a thermometer [shown as Formula 6 (BH90) in the APPENDIXES] that considers the effect of pressure. The ideal amphibole model has been criticized for its oversimplicity (e.g., Poli and Schmidt, 1992). In addition, temperatures were obtained for aluminous hornblendes coexisting with plagioclase, whereas erroneously high temperatures were obtained for garnet-amphibolites (Mengel and Rivers, 1991). Holland and Blundy (1994) found that withinand across-site interactions in amphiboles vary. Therefore, assuming a non-ideal amphibole, Holland and Blundy (1994) presented two geothermometers. One, which is presented as Formula $7\left(\mathrm{HB}_{1} 94\right)$ in the APPENDIXES, can be used for amphiboles coexisting with plagioclases in quartz-bearing rocks. The other, which is presented as Formula $8\left(\mathrm{HB}_{2} 94\right)$ in the APPENDIXES, can be used for amphiboles in rocks with or without quartz.

The present study aims to estimate the crystallization temperature and pressure of amphiboles in intermediate to basic rocks from the Almogholagh pluton by using some of the aforementioned methods. It also introduces intersection among geothermometers as a new method to estimate crystallization temperature and compares the results of this method with those of other methods.

\section{GEOLOGICAL SETTING}

Stocklin and Nabavi (1972) divided the Iranian plateau into eight segments: Zagros folding, Zagros thrusting, the Sanandaj-Sirjan Zone (SSZ), the Urumieh-Dokhtar magmatic arc (UDMA), central Iran, Alborz, Kopeh Dagh, and eastern Iran. The southwestern part of Iran was divided into three parallel NW-SE trending tectonic zones (Alavi, 1994), namely, the Zagros Thrust and Fold Belt, the SSZ, and the Tertiary-Quaternary UDMA (Fig. 1), which is an integral part of the Alpine-Himalayan orogenic system that resulted from Arabian-Central Iranian microplate continental collision after the subduction of the Neo-Tethys oceanic crust beneath Central Iran (Sengör, 1984). The SSZ is geographically subdivided into southern (S-SSZ) and northern (N-SSZ) parts by Eftekharnejad (1981) (Fig. 1). During the Paleozoic period, the SSZ was part of northeast Gondwanaland, separated from the Eurasian plate by the Paleo-Tethys ocean (Golonka, 2004). During the Middle to Late Triassic, coeval with the closure of the Palaeo-Tethys in the North, a rifting episode along the Zagros belt resulted in the opening of a new ocean called Neo-Tethys, and the SSZ was separated from the Arabian plate (Gondwanaland) as a part of Eurasia. Inception of subduction of Neo-Tethys was inferred to have occurred during the Late Triassic-Early

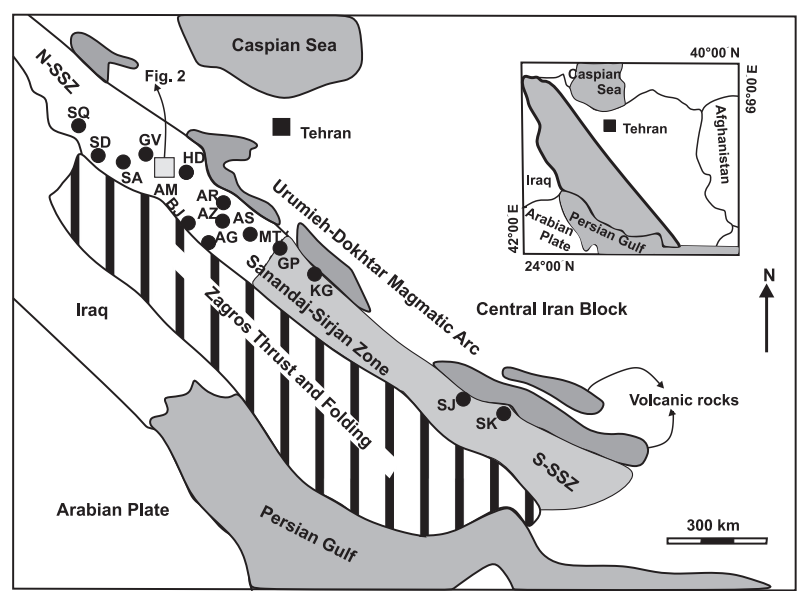

Figure 1. Geotectonic map showing the spatial distribution of Iranian southwestern tectonic units and major plutonic bodies, as well as the location of study area (square), in the Sanandaj-Sirjan Zone according to Stocklin and Nabavi (1972) and was modified by Sepahi et al. (2009). Abbreviations: SQ, Saqqez; SD, Sanandaj; GV, Ghorveh; AM (square as Fig. 2), Almogholagh; HD, Hamedan (Alvand); AR, Arak; AS, Astaneh; BJ, Boroujerd; AG, Aligudarz; AZ, Azna; MT, Muteh; GP, Golpaygan; KG, Kolah-Ghazi; SJ, Sirjan; and SK, Siah Kouh. Color version of Figures 1 is available online from http://doi.org/10.2465/ jmps. 151228 .

Jurassic (Berberian and Berberian, 1981). This phenomenon deformed rocks and some intrusive bodies in S-SSN (Late Triassic) and emplaced numerous intrusive bodies ranging from gabbro to granite in N-SSZ (Fig. 1). The ages of zircon $\mathrm{U}-\mathrm{Pb}$ imply that many SSZ granitoids were emplaced during the Middle Jurassic (e.g., Shahbazi et al., 2010), Middle to Late Cretaceous (e.g., Ghalamghash et al., 2009), and Early Eocene (Mazhari et al., 2009). Closure of the oceanic crust domain was marked by the obduction of ophiolites along the main Zagros thrust in the Late Cretaceous to Paleocene (Agard et al., 2005) and of some gabbroic bodies in the Upper Eocene to Oligo-Miocene, indicating that the final closure of the Neo-Tethys and collision of Arabian plate and Central Iran occurred during the Neogene period (Berberian and Berberian, 1981; Azizi and Moinevaziri, 2009).

The Almogholagh pluton forms part of a linear belt of N-SSZ intrusions in western Iran (Fig. 1). Country rocks, namely, the Hamedan phylites, comprise the Triassic-Jurassic metapelites (Mohajjel et al., 2003). Several regional and contact metamorphisms, including garnetmica schists, interbedded metavolcanics, marbles, and dolomitic limestones, occur around the pluton. Field observations revealed that Almogholagh intrusive rocks can be classified into three groups, namely, dark grey diorite or gabbroic diorite, grey-colored quartz syenite, and light-colored quartz monzonite. Other researchers have 
reported the presence of rocks, such as granite, quartz monzonite, monzonite, and monzodiorite, in this pluton (Shahbazi et al., 2015). Mohajjel and Izadi Kian (2007) have proven the occurrence of four deformations $\left(D_{1}-D_{4}\right)$ in the Almogholagh area that the pluton intruded during $\mathrm{D}_{2}$ deformation. Valizadeh and Zarian (1976), Shahbazi et al. (2015), and Amiri (2015) found that the ages of the Almogholagh masses are $144 \pm 17,95-138$, and $131 \pm 25$ Ma by using three-point $\mathrm{Rb}-\mathrm{Sr}$ whole-rock isochron, $\mathrm{U}-$ $\mathrm{Pb}$ and $\mathrm{U}-\mathrm{Th}-\mathrm{Pb}$ methods (for magmatic sphenes), and seven-point $\mathrm{Rb}-\mathrm{Sr}$ whole-rock isochron, respectively.

\section{SAMPLES AND METHODS}

In this study, approximately 80 samples were collected from the Almogholagh pluton during a 20-day operation. A total of 85 thin sections of these samples were prepared and studied using an optical microscope. Representative samples (22 samples) were selected to analyze wholerock geochemistry. Major and trace elements were analyzed through inductively coupled plasma-mass spectrometry equipped with an LF-200 system at the ACME Analytical Laboratories in Vancouver, Canada, and the results are presented by Amiri (2015). Different lithological diagrams were then drawn, and rock types were identified using the percentages of major oxides and GCDkit software. In addition, 16 square polished $1 \mathrm{~mm} \times 1 \mathrm{~mm} \times$ $0.5 \mathrm{~mm}$ disks were prepared for scanning elecron microscopy to study the mineral phases and to obtain back-scattered electron images. The samples were coated with a layer of $20 \mathrm{~nm}$-thick carbon by using the sputtering method and then studied under a MIRA3 field-emission scanning electron microscope.

Three thin polished sections of intermediate to basic rocks (Am48, Am47, and Am46) and acid rocks (Am23, Am07, and Am07-2) were prepared for microprobe analyses. The sampling site is shown in the lithological map (Fig. 2). We tried our best to obtain the less altered samples. The amphiboles and coexisting plagioclases in the sections were analyzed at the Iran Mineral Processing Research Center Laboratory by using Cameca SX 100 Electron Probe Micro-Analyzer, which was operated at a probe current of $20 \mathrm{nA}$ with an accelerating voltage of $20 \mathrm{kV}$. The counting duration is usually $35 \mathrm{~s}$ for the peak of each element. The electron beam diameter was set to $10 \mu \mathrm{m}$. The rims of amphibole and plagioclase crystals that are located close to one another were analyzed (e.g., Fig. 3c). A total of 44 points were analyzed, 20 of which belong to intermediate to basic rocks (Tables 1 and 2) and 24 belong to acid rocks (9 points in green amphiboles, 9 points in plagioclases coexisting with amphiboles, and 6 points in an association of euhedral

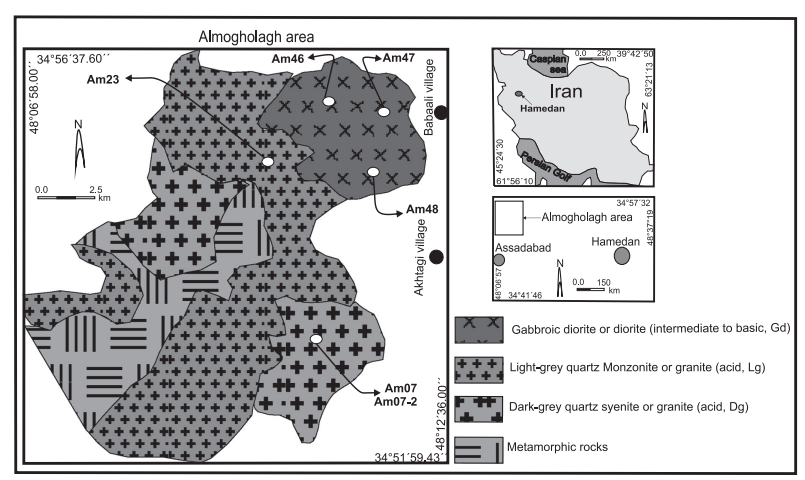

Figure 2. Locational and lithological map of the Almogholagh plutonic rocks. Color version of Figures 2 is available online from http://doi.org/10.2465/jmps. 151228 .

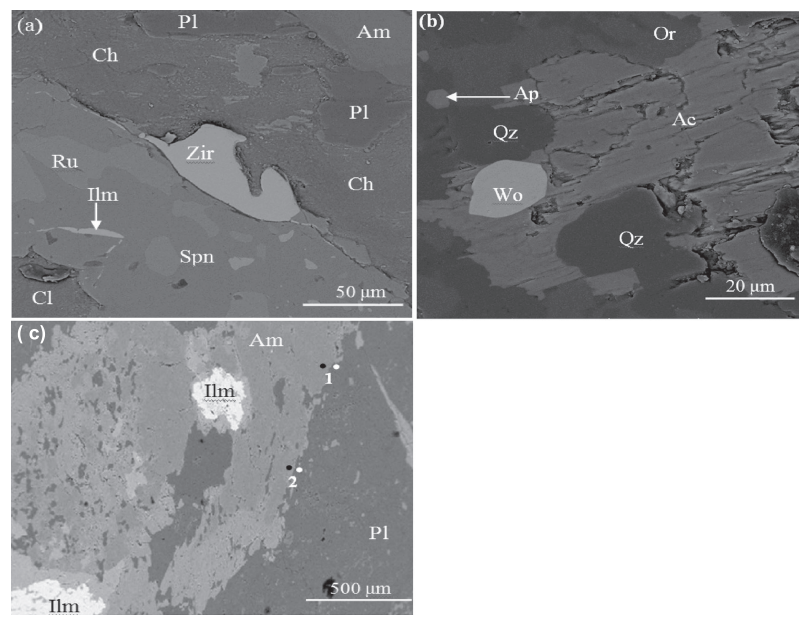

Figure 3. Backscattered electron images of minerals in (a), (b) acidic and (c) intermediate rocks. (a) Photograph of AM20 containing $\mathrm{Ch}$ and $\mathrm{Cl}$ secondary crystals. (b) Photograph of AM12 containing Ac secondary amphibole. (c) Photograph of AM48 containing Am and coexisting Pl. Abbreviations: Cl, $\mathrm{Zr}$-bearing clinochlore; $\mathrm{Ch}, \mathrm{Zr}$-Fe-bearing clinochlore; $\mathrm{Ru}$, rutile; Am, amphibole; Zir, zircon; Pl, plagioclase; Spn, sphene; Ilm, ilmenite; Ac, actinolite; Ap, apatite; Or, orthoclase; Qz, quartz; Wo, tungsten-bearing mineral. Symbols: the circles indicate the analyzed points (by EPMA). Color version of Figures 3 is available online from http://doi.org/10.2465/jmps.151228.

green-brown amphiboles lying near each other). Analytical data on plagioclases were calculated as atomic values of the elements based on eight atoms of oxygen and for intermediate to basic rocks are presented in Table 2. The $\mathrm{Fe}^{2+}$ and $\mathrm{Fe}^{3+}$ contents of the analyzed points in amphiboles were estimated by using the method described by Spear and Kimball (1984), and the structural formulas of the amphiboles were determined (Tables 1 and 3). The amphiboles were also classified and named using the structural formulas and the methods in IMA1997 (Leake et al., 1997) and IMA2004 (Leake et al., 2004). 
Table 1. Results of the microprobe analysis and the calculated atomic values of the amphiboles

\begin{tabular}{|c|c|c|c|c|c|c|c|c|c|c|}
\hline Spots & $\begin{array}{l}\mathrm{Am} \\
48-1\end{array}$ & $\begin{array}{l}\mathrm{Am} \\
48-2\end{array}$ & $\begin{array}{c}\mathrm{Am} \\
48-3\end{array}$ & $\begin{array}{c}\mathrm{Am} \\
48-4\end{array}$ & $\begin{array}{l}\mathrm{Am} \\
47-1\end{array}$ & $\begin{array}{l}\mathrm{Am} \\
47-2\end{array}$ & $\begin{array}{c}\mathrm{Am} \\
47-3\end{array}$ & $\begin{array}{l}\mathrm{Am} \\
46-1\end{array}$ & $\begin{array}{c}\mathrm{Am} \\
46-2\end{array}$ & $\begin{array}{c}\mathrm{Am} \\
46-3\end{array}$ \\
\hline $\mathrm{SiO}_{2}$ & 40.80 & 42.00 & 41.26 & 40.92 & 42.50 & 40.79 & 40.25 & 39.91 & 40.014 & 39.63 \\
\hline $\mathrm{TiO}_{2}$ & 0.465 & 0.430 & 0.523 & 0.393 & 0.440 & 0.254 & 0.250 & 0.275 & 0.245 & 0.196 \\
\hline $\mathrm{Al}_{2} \mathrm{O}_{3}$ & 12.13 & 11.27 & 12.01 & 11.54 & 11.24 & 12.38 & 13.11 & 13.64 & 12.75 & 13.15 \\
\hline $\mathrm{Fe}_{2} \mathrm{O}_{3}$ & - & - & - & - & - & - & - & - & - & - \\
\hline $\mathrm{FeO}$ & 20.62 & 19.69 & 19.95 & 19.42 & 19.13 & 20.63 & 21.33 & 23.64 & 22.74 & 21.796 \\
\hline $\mathrm{MnO}$ & 0.211 & 0.173 & 0.210 & 0.200 & 0.178 & 0.181 & 0.179 & 0.172 & 0.178 & 0.200 \\
\hline $\mathrm{MgO}$ & 8.240 & 9.197 & 8.806 & 8.964 & 9.881 & 8.707 & 7.870 & 7.525 & 8.202 & 7.426 \\
\hline $\mathrm{CaO}$ & 11.01 & 11.13 & 11.23 & 11.16 & 11.21 & 11.30 & 11.07 & 11.13 & 11.00 & 11.098 \\
\hline $\mathrm{Na}_{2} \mathrm{O}$ & 1.951 & 1.799 & 1.817 & 1.994 & 1.845 & 2.213 & 2.152 & 2.099 & 2.122 & 2.211 \\
\hline $\mathrm{K}_{2} \mathrm{O}$ & 1.100 & 0.878 & 0.894 & 0.871 & 0.789 & 0.993 & 0.991 & 1.328 & 1.072 & 1.319 \\
\hline Total & 96.52 & 96.56 & 96.70 & 95.47 & 97.21 & 97.46 & 97.21 & 99.64 & 98.32 & 97.02 \\
\hline \multicolumn{11}{|c|}{ The amphibole formulae base on corrected iron by help of Spear and Kimball (1984) method } \\
\hline $\mathrm{Si}$ & 6.27 & 6.396 & 6.288 & 6.323 & 6.397 & 6.204 & 6.152 & 5.990 & 6.052 & 6.115 \\
\hline $\mathrm{Ti}$ & 0.054 & 0.050 & 0.060 & 0.045 & 0.050 & 0.029 & 0.029 & 0.031 & 0.028 & 0.023 \\
\hline $\mathrm{Al}^{\mathrm{tat}}$ & 2.186 & 2.022 & 2.158 & 2.102 & 1.994 & 2.219 & 2.361 & 2.412 & 2.272 & 2.391 \\
\hline $\mathrm{Al}^{\mathrm{iv}}$ & 1.729 & 1.604 & 1.712 & 1.677 & 1.603 & 1.179 & 1.848 & 2.010 & 1.896 & 1.885 \\
\hline $\mathrm{Al}^{\mathrm{vi}}$ & 0.357 & 0.418 & 0.446 & 0.425 & 0.391 & 0.423 & 0.512 & 0.402 & 0.376 & 0.506 \\
\hline $\mathrm{Fe}^{3+}$ & 0.555 & 0.580 & 0.611 & 0.554 & 0.625 & 0.637 & 0.642 & 0.901 & 0.969 & 0.587 \\
\hline $\mathrm{Fe}^{2+}$ & 2.085 & 1.927 & 1.932 & 1.885 & 1.782 & 1.989 & 2.085 & 2.061 & 1.908 & 2.226 \\
\hline $\mathrm{Mn}$ & 0.028 & 0.022 & 0.027 & 0.026 & 0.023 & 0.023 & 0.023 & 0.022 & 0.023 & 0.026 \\
\hline $\mathrm{Mg}$ & 1.888 & 2.088 & 2.001 & 2.065 & 2.217 & 1.974 & 1.793 & 1.688 & 1.908 & 1.708 \\
\hline $\mathrm{Ca}^{-}$ & 1.813 & 1.817 & 1.834 & 1.848 & 1.808 & 1.841 & 1.813 & 1.790 & 1.783 & 1.835 \\
\hline $\mathrm{Na}^{+}$ & 0.582 & 0.531 & 0.537 & 0.597 & 0.538 & 0.652 & 0.638 & 0.610 & 0.622 & 0.661 \\
\hline $\mathrm{K}$ & 0.215 & 0.170 & 0.174 & 0.172 & 0.151 & 0.193 & 0.193 & 0.254 & 0.206 & 0.259 \\
\hline $\mathrm{OH}$ & 2.000 & 2.000 & 2.000 & 2.000 & 2.000 & 2.000 & 2.000 & 2.000 & 2.000 & 2.000 \\
\hline Sum Si & 6.271 & 6.396 & 6.288 & 6.323 & 6.397 & 6.204 & 6.152 & 5.990 & 6.052 & 6.116 \\
\hline Sum Al & 8.522 & 8.467 & 8.506 & 8.471 & 8.442 & 8.453 & 8.543 & 8.434 & 8.352 & 8.529 \\
\hline SumMg & 13.09 & 13.09 & 13.08 & 13.07 & 13.09 & 13.07 & 13.09 & 13.10 & 13.10 & 13.08 \\
\hline Sum Ca & 14.90 & 14.90 & 14.91 & 14.92 & 14.90 & 14.92 & 14.90 & 14.89 & 14.88 & 14.91 \\
\hline SumNa & 15.48 & 15.43 & 15.45 & 15.52 & 15.44 & 15.57 & 15.63 & 15.50 & 15.51 & 15.57 \\
\hline Sum K & 15.70 & 15.65 & 15.62 & 15.69 & 15.59 & 15.76 & 15.73 & 15.75 & 15.71 & 15.88 \\
\hline
\end{tabular}

Upon ensuring that the analyzed points are not subsolidus amphiboles (actinolite), we calculated the crystallization pressure and temperature by using some of the aforementioned methods. The results obtained by using these methods were compared. Finally, geothermometer intersection was used as a method to estimate the temperature of magma during the crystallization of the analyzed amphiboles, and the results obtained by using this method were compared with those of existing methods.

\section{RESULTS AND DISCUSSIONS}

\section{Petrography}

On the basis of the results of the observation of the thin sections, intrusive rocks were classified into two groups. The first group is labeled as gabbroic diorite and diorite in the lithological map (Gd in Fig. 2); this group includes dark, granular, and medium to fairly coarse grained rocks that contain light green to olive green amphiboles $(\sim 30$ $35 \%)$, alkalic feldspar and plagioclase $(\sim 55-60 \%)$, fine- grained quartz in the field $(\sim 3-10 \%)$, rhombic sphenes as accessory minerals $(\sim 2 \%)$, and rhombic magnetites and ilmenites as conspicuous opaque minerals $(\sim 4 \%)$. Clinopyroxene is rarely observed in some samples. The plagioclases occur as euhedral to subhedral without zoning crystals, although they display polysynthetic twinning and generally an extinction angle of lower than $35^{\circ}$. The plagioclases are mainly of oligoclase or andesine types, and some of them appear as albite or labradorite. On the basis of the diagram prepared by Middlemost (1994), the Am-46, Am-47, and Am-48 samples are identified either as quartz diorite or monzodiorite rocks.

The second group (acid rocks) includes light- or grey-colored porphyritic rocks that contain feldspars and amphiboles as their coarse minerals. This group contains amphiboles $(\sim 15-20 \%)$, alkalic feldspars and plagioclases $(\sim 50-55 \%)$, leucoxene + ilmenite + sphene + magnetite $(\sim 10 \%)$, and fine-grained quartz $(\sim 15-25 \%)$. Shorl-F tourmaline is rarely observed in some thin sections. Plagioclases in acidic rocks with an extinction angle between 12 and $20^{\circ}$ correspond to albite or oligoclase 
Table 2. Results of the microprobe analysis and the calculated atomic values of the plagioclases

\begin{tabular}{lcccccccccc}
\hline Spots & $\mathrm{Am}$ & $\mathrm{Am}$ & $\mathrm{Am}$ & $\mathrm{Am}$ & $\mathrm{Am}$ & $\mathrm{Am}$ & $\mathrm{Am}$ & $\mathrm{Am}$ & $\mathrm{Am}$ & $\mathrm{Am}$ \\
& $48-1$ & $48-2$ & $48-3$ & $48-4$ & $47-1$ & $47-2$ & $47-3$ & $46-1$ & $46-2$ & $46-3$ \\
\hline $\mathrm{SiO}_{2}$ & 62.77 & 62.94 & 68.55 & 63.08 & 59.46 & 63.61 & 64.42 & 67.15 & 60.77 & 60.10 \\
$\mathrm{TiO}_{2}$ & 0.000 & 0.019 & 0.008 & 0.021 & 0.055 & 0.000 & 0.010 & 0.000 & 0.012 & 0.029 \\
$\mathrm{Al}_{2} \mathrm{O}_{3}$ & 22.84 & 22.65 & 20.26 & 22.51 & 23.83 & 22.22 & 22.37 & 21.67 & 23.81 & 24.13 \\
$\mathrm{FeO}$ & 0.114 & 0.158 & 0.057 & 0.080 & 2.389 & 0.159 & 0.052 & 0.049 & 0.043 & 0.689 \\
$\mathrm{MnO}$ & 0.000 & 0.000 & 0.000 & 0.010 & 0.007 & 0.000 & 0.000 & 0.000 & 0.003 & 0.016 \\
$\mathrm{MgO}$ & 0.034 & 0.031 & 0.055 & 0.008 & 0.000 & 0.259 & 0.052 & 0.144 & 0.429 & 0.083 \\
$\mathrm{CaO}$ & 4.573 & 3.482 & 0.978 & 3.878 & 6.028 & 3.706 & 3.532 & 2.007 & 5.652 & 6.000 \\
$\mathrm{Na}_{2} \mathrm{O}$ & 3.397 & 9.391 & 10.12 & 9.797 & 8.310 & 10.01 & 10.18 & 9.645 & 8.971 & 8.725 \\
$\mathrm{~K}_{2} \mathrm{O}$ & 0.042 & 0.331 & 0.076 & 0.060 & 0.052 & 0.044 & 0.051 & 0.150 & 0.081 & 0.143 \\
$\mathrm{P}_{2} \mathrm{O}_{5}$ & 0.000 & 0.031 & 0.008 & 0.000 & 0.140 & 0.000 & 0.000 & 0.013 & 0.000 & 0.016 \\
$\mathrm{Total}$ & 99.76 & 99.13 & 100.1 & 99.54 & 100.4 & 100.0 & 100.7 & 100.8 & 99.77 & 99.93 \\
$\mathrm{Si}$ & 2.790 & 2.817 & 2.976 & 2.809 & 2.261 & 2.817 & 2.823 & 2.904 & 2.712 & 2.691 \\
$\mathrm{Ti}$ & 0.000 & 0.001 & 0.000 & 0.000 & 0.002 & 0.000 & 0.000 & 0.000 & 0.000 & 0.000 \\
$\mathrm{Al}$ & 1.196 & 1.188 & 1.024 & 1.181 & 1.292 & 1.159 & 1.164 & 1.083 & 1.255 & 0.276 \\
$\mathrm{Fe}$ & 0.004 & 0.005 & 0.003 & 0.003 & 0.088 & 0.005 & 0.003 & 0.003 & 0.003 & 0.027 \\
$\mathrm{Mn}$ & 0.000 & 0.000 & 0.000 & 0.000 & 0.000 & 0.000 & 0.000 & 0.000 & 0.000 & 0.000 \\
$\mathrm{Mg}$ & 0.003 & 0.002 & 0.003 & 0.000 & 0.000 & 0.016 & 0.003 & 0.010 & 0.029 & 0.005 \\
$\mathrm{Ca}$ & 0.219 & 0.162 & 0.044 & 0.182 & 0.297 & 0.176 & 0.165 & 0.097 & 0.271 & 0.288 \\
$\mathrm{Na}^{+}$ & 0.810 & 0.800 & 0.921 & 0.845 & 0.754 & 0.859 & 0.845 & 0.905 & 0.778 & 0.759 \\
$\mathrm{~K}$ & 0.001 & 0.019 & 0.005 & 0.003 & 0.003 & 0.003 & 0.005 & 0.010 & 0.005 & 0.011 \\
$\mathrm{P}$ & 0.000 & 0.000 & 0.000 & 0.000 & 0.003 & 0.000 & 0.000 & 0.000 & 0.000 & 0.000 \\
$\mathrm{An}$ & 0.211 & 0.167 & 0.047 & 0.177 & 0.287 & 0.169 & 0.158 & 0.096 & 0.258 & 0.272 \\
$\mathrm{Ab}$ & 0.790 & 0.814 & 0.940 & 0.820 & 0.711 & 0.828 & 0.836 & 0.894 & 0.737 & 0.718 \\
$\mathrm{Or}$ & 0.003 & 0.018 & 0.004 & 0.003 & 0.003 & 0.003 & 0.005 & 0.010 & 0.005 & 0.010 \\
\hline
\end{tabular}

type. Two types of amphiboles are observed. One is a green-brown euhedral early-formed amphibole, and the other is a green subhedral late-formed amphibole. Acid rocks are divided into two sub-groups, namely, light-grey quartz monzonite (Lg in Fig. 2) and dark-grey quartz syenite (Dg in Fig. 2). The quartz syenite has finer grains, and their amphiboles and opaque minerals (sphenes and magnetites) are scattered, whereas the amphiboles and opaque minerals in the quartz monzonite exhibit clots (microglomeroporphyritic texture). The granophyric texture of quartz monzonite suggests that the quartz was subjected to a sudden decrease in pressure and probably could have come near the surface during the last stage of formation. Secondary crystals such as actinolite, clinochlore, and leucoxene formed in acidic rocks. These minerals show that active fluids attacked amphiboles and plagioclases in acidic rocks (Figs. 3a and 3b). On the basis of the diagram prepared by Middlemost (1994), the Am23, Am-07, and Am-07-2 samples are identified either as quartz monzonite or quartz syenite.

In some petrologic diagrams (Cox et al., 1979; De La Roche et al., 1980; Debon and Lefort, 1983; Middlemost, 1994), the following various rocks are identified as follows: quartz-syenite, quartz-monzonite, granite, quartzdiorite, diorite, gabbroic diorite, and gabbro, which are generally divided into two groups as mentioned above.

\section{Geochemistry of pluton}

In the diagram prepared by Cox et al. (1979), Almogholagh intrusive rocks are located beside the separating border of the alkaline series from the subalkaline and tholeiitic series. In the diagram of Irvine and Baragar (1971), the intermediate to basic rocks are placed in the calc-alkaline series. The results of a systematic geochemical study based on various diagrams show that the intermediate to basic rocks are calc-alkaline I-type intrusions and associated with late or post-collision stage (i.e., III-type of Harris et al., 1986). In addition, the acid rocks are $\mathrm{A}_{2}-$ type intrusions and associated with late or post-collision stage. In $\mathrm{Ce}$ and $\mathrm{Nb}$ versus $10000 \mathrm{Ga} / \mathrm{Al}$ diagrams of Whalen et al. (1987), all quartz monzonite and quartz syenite samples (acid rocks) plot in the A-type granitoids field, whereas Almogholagh intermediate to basic (gabbroic diorite) samples plot in the I- and S-type granitoids field. The Almogholagh intrusions exhibit the following characteristics: $\varepsilon \mathrm{Sr}>0, \varepsilon \mathrm{Nd}>0, \mathrm{Ba} / \mathrm{La}>3,(\mathrm{Rb} / \mathrm{Ba})_{\mathrm{N}}>$ 1; distinctive spiked pattern with peaks at $\mathrm{Rb}, \mathrm{Ba}, \mathrm{Th}, \mathrm{K}$, $\mathrm{Sr}$, and $\mathrm{Zr}$; light-REE enriched pattern; low abundance of elements with high ionic potential (e.g., Dy, Y, Yb, Lu, and $\mathrm{Sm}$ ); and high aboundance of $\mathrm{Nb}$, with weak negative anomalies of it (Fig. 4). These properties are apparently a characteristic of subduction-related magmas, demonstrating the involvement of subduction zone fluids, 
Table 3. Names and structural formulas of the analyzed points (in amphiboles)

\begin{tabular}{|c|c|c|}
\hline Spots & $\begin{array}{ll} & \text { Structural Formula } \\
\end{array}$ & Nomenclatures \\
\hline Am48-1 & $\left(\mathrm{Na}_{0.36} \mathrm{~K}_{0.215}\right)_{0.575}\left(\mathrm{Ca}_{1.778} \mathrm{Na}_{0.222}\right)_{2.0}\left(\mathrm{Al}_{0.357} \mathrm{Ti}_{0.535} \mathrm{Fe}^{3+}{ }_{0.555} \mathrm{Mg}_{1.888} \mathrm{Fe}^{2+}{ }_{2.085} \mathrm{Mn}_{0.027}\right.$ & Hastingsite \\
\hline Am48-2 & $\begin{array}{l}\left.\mathrm{Ca}_{0.035}\right)_{5.0}\left(\mathrm{Si}_{6.271} \mathrm{Al}_{1.729}\right)_{8.0} \mathrm{O}_{22}(\mathrm{OH})_{2 .} \\
\left(\mathrm{Na}_{0.383} \mathrm{~K}_{0.17}\right)_{0.553}\left(\mathrm{Mn}_{0.022} \mathrm{Fe}^{2+}{ }_{0.013} \mathrm{Ca}_{1.817} \mathrm{Na}_{0.148}\right)_{2.0}\left(\mathrm{Al}_{0.418} \mathrm{Ti}_{0.05} \mathrm{Fe}^{3+}{ }_{0.58} \mathrm{Mg}_{2.088} \mathrm{Fe}^{2+}{ }_{1.914}\right)_{5.0} \\
\left(\mathrm{Si}_{6.396} \mathrm{Al}_{1.604}\right)_{8.0} \mathrm{O}_{22}(\mathrm{OH})_{2}\end{array}$ & Magnesiohastingsite \\
\hline Am48-3 & $\begin{array}{l}\left(\mathrm{Na}_{0.447} \mathrm{~K}_{0.174}\right)_{0.621}\left(\mathrm{Mn}_{0.027} \mathrm{Fe}_{0.049} \mathrm{Ca}_{1.834} \mathrm{Na}_{0.09}\right)_{2.0}\left(\mathrm{Al}_{0.446} \mathrm{Ti}_{0.06} \mathrm{Fe}^{3+}{ }_{0.611} \mathrm{Mg}_{2.001} \mathrm{Fe}^{2+}{ }_{1.883}\right)_{5.0} \\
\left(\mathrm{Si}_{6.288} \mathrm{Al}_{1.712}\right)_{8.0} \mathrm{O}_{22}(\mathrm{OH})_{2}\end{array}$ & Magnesiohastingsite \\
\hline Am48-4 & $\begin{array}{l}\left(\mathrm{Na}_{0.445} \mathrm{~K}_{0.172}\right)_{0.617}\left(\mathrm{Ca}_{1.848} \mathrm{Na}_{0.152}\right)_{2.0}\left(\mathrm{Al}_{0.425} \mathrm{Ti}_{0.045} \mathrm{Fe}^{3+}{ }_{0.554} \mathrm{Mg}_{2.065} \mathrm{Fe}^{2+}{ }_{1.885} \mathrm{Mn}_{0.026}\right)_{5.0} \\
\left(\mathrm{Si}_{6.323} \mathrm{Al}_{1.677}\right)_{8.0} \mathrm{O}_{22}(\mathrm{OH})_{2}\end{array}$ & Magnesiohastingsite \\
\hline Am47-1 & $\begin{array}{l}\left(\mathrm{Na}_{0.434} \mathrm{~K}_{0.151}\right)_{0.585}\left(\mathrm{Mn}_{0.023} \mathrm{Fe}^{2+}{ }_{0.065} \mathrm{Ca}_{1.808} \mathrm{Na}_{0.104}\right)_{2.0}\left(\mathrm{Al}_{0.391} \mathrm{Ti}_{0.05} \mathrm{Fe}^{3+}{ }_{0.625} \mathrm{Mg}_{2.217} \mathrm{Fe}^{2+}{ }_{1.717}\right)_{5.0} \\
\left(\mathrm{Si}_{6.397} \mathrm{Al}_{1.603}\right)_{8.0} \mathrm{O}_{22}(\mathrm{OH})_{2 .}\end{array}$ & Magnesiohastingsite \\
\hline Am47-2 & $\begin{array}{l}\left(\mathrm{Na}_{0.565} \mathrm{~K}_{0.193}\right)_{0.758}\left(\mathrm{Mn}_{0.023} \mathrm{Fe}^{2+}{ }_{0.049} \mathrm{Ca}_{1.841} \mathrm{Na}_{0.087}\right)_{2.0}\left(\mathrm{Al}_{0.423} \mathrm{Ti}_{0.029} \mathrm{Fe}^{3+}{ }_{0.637} \mathrm{Mg}_{1.974} \mathrm{Fe}^{2+}{ }_{1.937}\right)_{5.0} \\
\left(\mathrm{Si}_{6.204} \mathrm{Al}_{1.796}\right)_{8.0} \mathrm{O}_{22}(\mathrm{OH})_{2}\end{array}$ & Hastingsite \\
\hline Am47-3 & $\begin{array}{l}\left(\mathrm{Na}_{0.535} \mathrm{~K}_{0.193}\right)_{0.728}\left(\mathrm{Mn}_{0.023} \mathrm{Fe}^{2+}{ }_{0.061} \mathrm{Ca}_{1.813} \mathrm{Na}_{0.103}\right)_{2.0}\left(\mathrm{Al}_{0.512} \mathrm{Ti}_{0.029} \mathrm{Fe}^{3+}{ }_{0.642} \mathrm{Mg}_{1.793} \mathrm{Fe}^{2+}{ }_{2.024}\right)_{5.0} \\
\left(\mathrm{Si}_{6.152} \mathrm{Al}_{1.848}\right)_{8.0} \mathrm{O}_{22}(\mathrm{OH})_{2}\end{array}$ & Hastingsite \\
\hline Am46-1 & $\begin{array}{l}\left(\mathrm{Na}_{0.505} \mathrm{~K}_{0.254}\right)_{0.759}\left(\mathrm{Mn}_{0.022} \mathrm{Fe}^{2+}{ }_{0.083} \mathrm{Ca}_{1.79} \mathrm{Na}_{0.105}\right)_{2.0}\left(\mathrm{Al}_{0.402} \mathrm{Ti}_{0.031} \mathrm{Fe}^{3+}{ }_{0.901} \mathrm{Mg}_{1.688} \mathrm{Fe}^{2+}{ }_{1.978}\right)_{5.0} \\
\left(\mathrm{Si}_{5.99} \mathrm{Al}_{2.01}\right)_{8.0} \mathrm{O}_{22}(\mathrm{OH})_{2}\end{array}$ & Hastingsite \\
\hline Am46-2 & $\begin{array}{l}\left(\mathrm{Na}_{0.617} \mathrm{~K}_{0.206}\right)_{0.823}\left(\mathrm{Mn}_{0.023} \mathrm{Fe}^{2+}{ }_{0.189} \mathrm{Ca}_{1.783} \mathrm{Na}_{0.005}\right)_{2.0}\left(\mathrm{Al}_{0.376} \mathrm{Ti}_{0.028} \mathrm{Fe}^{3+}{ }_{0.969} \mathrm{Mg}_{1.908} \mathrm{Fe}^{2+}{ }_{1.719}\right)_{5.0} \\
\left(\mathrm{Si}_{6.052} \mathrm{Al}_{1.896}\right)_{8.0} \mathrm{O}_{22}(\mathrm{OH})_{2}\end{array}$ & Magnesiohastingsite \\
\hline Am46-3 & $\begin{array}{l}\left(\mathrm{Na}_{0.572} \mathrm{~K}_{0.259}\right)_{0.831}\left(\mathrm{Mn}_{0.026} \mathrm{Fe}^{2+}{ }_{0.05} \mathrm{Ca}_{1.835} \mathrm{Na}_{0.089}\right)_{2.0}\left(\mathrm{Al}_{0.506} \mathrm{Ti}_{0.023} \mathrm{Fe}^{3+}{ }_{0.587} \mathrm{Mg}_{1.708} \mathrm{Fe}^{2+}{ }_{2.176}\right)_{5.0} \\
\left(\mathrm{Si}_{6.115} \mathrm{Al}_{1.885}\right)_{8.0} \mathrm{O}_{22}(\mathrm{OH})_{2}\end{array}$ & Hastingsite \\
\hline Am23-1 & $\begin{array}{l}\left(\mathrm{Na}_{0.111} \mathrm{~K}_{0.036}\right)_{0.147}\left(\mathrm{Mn}_{0.04} \mathrm{Ca}_{1.911} \mathrm{Na}_{0.049}\right)_{2.0}\left(\mathrm{Al}_{0.113} \mathrm{Ti}_{0.006} \mathrm{Fe}^{3+}{ }_{0.101} \mathrm{Mg}_{3.208} \mathrm{Fe}^{2+}{ }_{1.568} \mathrm{Mn}_{0.004}\right)_{5.0} \\
\left(\mathrm{Si}_{7.715} \mathrm{Al}_{0.285}\right)_{8.0} \mathrm{O}_{22}(\mathrm{OH})_{2}\end{array}$ & Actinolite \\
\hline Am23-2 & $\begin{array}{l}\left(\mathrm{Ca}_{0.076} \mathrm{Na}_{0.189} \mathrm{~K}_{0.033}\right)_{0.298}\left(\mathrm{Mn}_{0.054} \mathrm{Fe}^{2+}{ }_{0.179} \mathrm{Ca}_{1.767}\right)_{2.0}\left(\mathrm{Al}_{0.121} \mathrm{Ti}_{0.006} \mathrm{Fe}^{3+}{ }_{0.178} \mathrm{Mg}_{3.031} \mathrm{Fe}^{2+}{ }_{1.664}\right)_{5.0} \\
\left(\mathrm{Si}_{7.814} \mathrm{Al}_{0.186}\right)_{8.0} \mathrm{O}_{22}(\mathrm{OH})_{2}\end{array}$ & Actinolite \\
\hline Am23-3 & $\begin{array}{l}\left(\mathrm{Na}_{0.102} \mathrm{~K}_{0.024}\right)_{0.126}\left(\mathrm{Mn}_{0.060} \mathrm{Ca}_{1.932} \mathrm{Na}_{0.008}\right)_{2.0}\left(\mathrm{Ti}_{0.003} \mathrm{Fe}^{3+}{ }_{0.069} \mathrm{Mg}_{3.102} \mathrm{Fe}^{2+}{ }_{1.826}\right)_{5.0} \\
\left(\mathrm{Si}_{7.803} \mathrm{Al}_{0.197}\right)_{8.0} \mathrm{O}_{22}(\mathrm{OH})_{2}\end{array}$ & Actinolite \\
\hline Am23-4 & $\begin{array}{l}\left(\mathrm{Na}_{0.088} \mathrm{~K}_{0.017}\right)_{0.105}\left(\mathrm{Fe}^{2+}{ }_{0.002} \mathrm{Mn}_{0.057} \mathrm{Ca}_{1.92} \mathrm{Na}_{0.021}\right)_{2.0}\left(\mathrm{Al}_{0.009} \mathrm{Ti}_{0.004} \mathrm{Fe}^{3+}{ }_{0.085} \mathrm{Mg}_{3.109} \mathrm{Fe}^{2+}{ }_{1.793}\right)_{5.0} \\
\left(\mathrm{Si}_{7.820} \mathrm{Al}_{0.18}\right)_{8.0} \mathrm{O}_{22}(\mathrm{OH})_{2}\end{array}$ & Actinolite \\
\hline Am23-5 & $\begin{array}{l}\left(\mathrm{Na}_{0.053} \mathrm{~K}_{0.019}\right)_{0.072}\left(\mathrm{Mn}_{0.046} \mathrm{Ca}_{1.896} \mathrm{Na}_{0.058}\right)_{2.0}\left(\mathrm{Al}_{0.055} \mathrm{Ti}_{0.002} \mathrm{Fe}^{3+}{ }_{0.124} \mathrm{Mg}_{3.09} \mathrm{Fe}^{2+}{ }_{1.718} \mathrm{Mn}_{0.011}\right)_{5.0} \\
\left(\mathrm{Si}_{7.842} \mathrm{Al}_{0.158}\right)_{8.0} \mathrm{O}_{22}(\mathrm{OH})_{2}\end{array}$ & Actinolite \\
\hline Am07-1 & $\begin{array}{l}\left(\mathrm{Ca}_{0.028} \mathrm{Na}_{0.121} \mathrm{~K}_{0.025}\right)_{0.174}\left(\mathrm{Fe}^{2+}{ }_{0.052} \mathrm{Mn}_{0.046} \mathrm{Ca}_{1.902}\right)_{2.0}\left(\mathrm{Al}_{0.009} \mathrm{Ti}_{0.005} \mathrm{Mg}_{2.862} \mathrm{Fe}_{2.124}\right)_{5.0} \\
\left(\mathrm{Si}_{7.776} \mathrm{Al}_{0.224}\right)_{8.0} \mathrm{O}_{22}(\mathrm{OH})_{2}\end{array}$ & Actinolite \\
\hline Am07-2 & $\begin{array}{l}\left(\mathrm{Na}_{0.097} \mathrm{~K}_{0.097}\right)_{0.194}\left(\mathrm{Fe}^{2+}{ }_{0.037} \mathrm{Mn}_{0.048} \mathrm{Ca}_{1.767} \mathrm{Na}_{0.148}\right)_{2.0}\left(\mathrm{Al}_{0.299} \mathrm{Ti}_{0.018} \mathrm{Fe}^{3+}{ }_{0.0} \mathrm{Mg}_{2.765} \mathrm{Fe}^{2+}{ }_{1.918}\right)_{5.0} \\
\left(\mathrm{Si}_{7.861} \mathrm{Al}_{0.139}\right)_{8.0} \mathrm{O}_{22}(\mathrm{OH})_{2}\end{array}$ & Actinolite \\
\hline Am07-3 & $\begin{array}{l}\left(\mathrm{K}_{0.073}\right)_{0.073}\left(\mathrm{Fe}^{2+}{ }_{0.042} \mathrm{Mn}_{0.047} \mathrm{Ca}_{1.812} \mathrm{Na}_{0.094}\right)_{1.995}\left(\mathrm{Al}_{0.163} \mathrm{Ti}_{0.019} \mathrm{Fe}^{3+}{ }_{0.018} \mathrm{Mg}_{3.321} \mathrm{Fe}^{2+}{ }_{1.48}\right) \\
\left(\mathrm{Si}_{7.832} \mathrm{Al}_{0.168}\right)_{8.0} \mathrm{O}_{22}(\mathrm{OH})_{2}\end{array}$ & Actinolite \\
\hline Am07-4 & $\begin{array}{l}\left(\mathrm{Ca}_{0.087} \mathrm{Na}_{0.071} \mathrm{~K}_{0.092}\right)_{0.25}\left(\mathrm{Fe}^{2+}{ }_{0.2} \mathrm{Mn}_{0.093} \mathrm{Ca}_{1.707}\right)_{2.0}\left(\mathrm{Al}_{0.122} \mathrm{Ti}_{0.092} \mathrm{Fe}^{3+}{ }_{0.10} \mathrm{Mg}_{3.169} \mathrm{Fe}^{2+}{ }_{1.517}\right)_{5.0} \\
\left(\mathrm{Si}_{7.651} \mathrm{Al}_{0.349}\right)_{8.0} \mathrm{O}_{22}(\mathrm{OH})_{2}\end{array}$ & Actinolite \\
\hline Am07-2-1 & $\begin{array}{l}\left(\mathrm{Ca}_{0.214} \mathrm{~K}_{0.02}\right)_{0.216}\left(\mathrm{Ca}_{2.0}\right)_{2.0}\left(\mathrm{Al}_{1.88} \mathrm{Ti}_{0.005} \mathrm{Mg}_{0.064} \mathrm{Fe}^{2+}{ }_{1.453} \mathrm{Mn}_{0.022} \mathrm{Ca}_{1.576}\right)_{5.0} \\
\left(\mathrm{Si}_{5.681} \mathrm{Al}_{2.319}\right)_{8.0} \mathrm{O}_{22}(\mathrm{OH})_{2}\end{array}$ & $\begin{array}{l}\text { Alumino- } \\
\text { Ferrotschermakite }\end{array}$ \\
\hline Am07-2-2 & $\begin{array}{l}\left(\mathrm{Ca}_{0.197} \mathrm{Na}_{0.003}\right)_{0.2}\left(\mathrm{Ca}_{2.0}\right)_{2.0}\left(\mathrm{Al}_{1.83} \mathrm{Ti}_{0.006} \mathrm{Mg}_{0.015} \mathrm{Fe}^{2+}{ }_{1.534} \mathrm{Mn}_{0.017} \mathrm{Ca}_{1.598}\right)_{5.0} \\
\left(\mathrm{Si}_{5.73} \mathrm{Al}_{2.27}\right)_{8.0} \mathrm{O}_{22}(\mathrm{OH})_{2}\end{array}$ & $\begin{array}{l}\text { Alumino- } \\
\text { Ferrotschermakite }\end{array}$ \\
\hline Am07-2-3 & $\begin{array}{l}\left(\mathrm{Ca}_{0.376} \mathrm{~K}_{0.003}\right)_{0.379}\left(\mathrm{Ca}_{2.0}\right)_{2.0}\left(\mathrm{Al}_{1.427} \mathrm{Ti}_{0.003} \mathrm{Mg}_{0.004} \mathrm{Fe}^{2+}{ }_{2.06} \mathrm{Mn}_{0.007} \mathrm{Ca}_{1.502}\right)_{5.0} \\
\left(\mathrm{Si}_{5.806} \mathrm{Al}_{2.194}\right)_{8.0} \mathrm{O}_{22}(\mathrm{OH})_{2}\end{array}$ & $\begin{array}{l}\text { Alumino- } \\
\text { Ferrotschermakite }\end{array}$ \\
\hline Am07-2-4 & $\begin{array}{l}\left(\mathrm{Ca}_{0.277} \mathrm{Na}_{0.015}\right)_{0.292}\left(\mathrm{Ca}_{2.0}\right)_{2.0}\left(\mathrm{Al}_{1.707} \mathrm{Ti}_{0.001} \mathrm{Fe}^{2+}{ }_{1.754} \mathrm{Mn}_{0.071} \mathrm{Ca}_{1.467}\right)_{5.0} \\
\left(\mathrm{Si}_{5.724} \mathrm{Al}_{2.276}\right)_{8.0} \mathrm{O}_{22}(\mathrm{OH})_{2}\end{array}$ & $\begin{array}{l}\text { Alumino- } \\
\text { Ferrotschermakite }\end{array}$ \\
\hline Am07-2-5 & $\begin{array}{l}\left(\mathrm{Ca}_{0.37} \mathrm{Na}_{0.01}\right)_{0.38}\left(\mathrm{Ca}_{2.0}\right)_{2.0}\left(\mathrm{Al}_{1.5} \mathrm{Ti}_{0.003} \mathrm{Mg}_{0.038} \mathrm{Fe}^{2+}{ }_{2.007} \mathrm{Mn}_{0.023} \mathrm{Ca}_{1.429}\right)_{5.0} \\
\left(\mathrm{Si}_{5.743} \mathrm{Al}_{2.257}\right)_{8.0} \mathrm{O}_{22}(\mathrm{OH})_{2}\end{array}$ & $\begin{array}{l}\text { Alumino- } \\
\text { Ferrotschermakite }\end{array}$ \\
\hline Am07-2-6 & $\begin{array}{l}\left(\mathrm{Ca}_{0.418}\right)_{0.418}\left(\mathrm{Ca}_{2.0}\right)_{2.0}\left(\mathrm{Al}_{1.533} \mathrm{Mg}_{0.008} \mathrm{Fe}^{2+}{ }_{2.017} \mathrm{Mn}_{0.032} \mathrm{Ca}_{1.41}\right)_{5.0} \\
\left(\mathrm{Si}_{5.776} \mathrm{Al}_{2.224}\right)_{8.0} \mathrm{O}_{22}(\mathrm{OH})_{2}\end{array}$ & $\begin{array}{l}\text { Alumino- } \\
\text { Ferrotschermakite }\end{array}$ \\
\hline
\end{tabular}

high flux of mantle-derived halogen-rich volatiles, and contamination within the crust during the petrogenesis of Almogholagh intrusions (Amiri, 2015).

The acid rocks generally contain high concentrations of $\mathrm{Na}_{2} \mathrm{O}+\mathrm{K}_{2} \mathrm{O}(9.48 \%)$, Sn (6.9 ppm), Ga (22.7 ppm), Ce (8.5 ppm), and Y (57 ppm). The average con- centration of $\mathrm{Zr}$ in both acidic and intermediate to basic rocks is considerably high, 589 and 165 ppm, respectively, and shows a distinctive spiked peak in spider diagrams (Fig. 4). When large quantities of fluorine and/or chlorine exist in the environment where magma is produced and crystallized (e.g., the presence of Shorl-F tourmaline in 


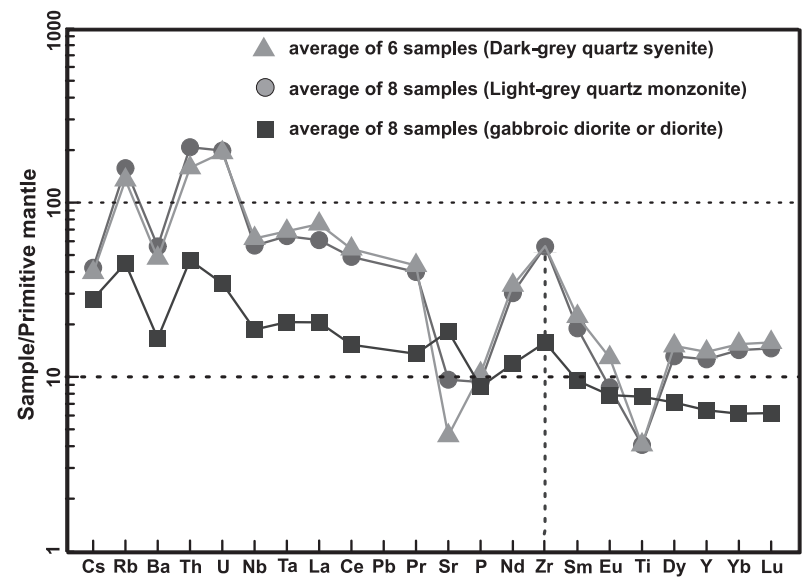

Figure 4. Primitive mantle-normalized element distributions for the intrusive rocks obtained from the Almogholagh pluton (the primitive mantle factors are based from those reported by McDonough and Sun, 1995). Color version of Figures 4 is available online from http://doi.org/10.2465/jmps. 151228 .

Almogholag pluton), the $\mathrm{F}$ and $\mathrm{C} 1$ in the magma distort the aluminosilicate framework, providing binding sites for the highly charged cations and stabilize the complexes of large, highly charged metal ions (e.g., Zr). An excess of alkalis over alumina and high $\mathrm{F}$ in the melt inhibits zircon formation and promotes the formation of zircono-fluoride melt complexes (Whalen et al. 1987). This phenomenon leads to the enrichment of $\mathrm{Zr}$ in the magma resulting from the melting of the parent rock, and $\mathrm{Zr}$ increases substantially during fractional crystallization. Harris and Marriner (1980) used the high flux of mantle-derived halogen-rich volatiles to induce melting and to provide a high concentration of alkalis and highfield-strength elements.

\section{Chemistry of amphiboles}

The microprobe can not distinguish between $\mathrm{Fe}^{2+}$ and $\mathrm{Fe}^{3+}$. Consequently, analyses are usually in terms of total iron, recorded as $\mathrm{FeO}$. However, $\mathrm{Fe}^{2+}$ and $\mathrm{Fe}^{3+}$ occupy different types of cation sites in amphiboles, and the amount of $\mathrm{Fe}^{3+}$ in an amphibole is critical in determining the A-site occupancy (Spear and Kimball, 1984). Therefore, the amounts of $\mathrm{Fe}^{2+}$ and $\mathrm{Fe}^{3+}$ were calculated by using a method employed in IMA to determine the structural formula of amphiboles. The aforementioned method was used to determine the structural formulas of the analyzed points (Table 3 ). To identify and determine the amphibole type in accordance with the instructions of IMA, we established diagram 5a on which the analyzed points were subsequently plotted. All of the samples were placed in the calcic amphibole group. Many researchers suggested that the presence of calcic amphiboles in granitoid rocks indicates that these rocks belong to I-type magma (e.g., Chappell and White, 1974). In addition,

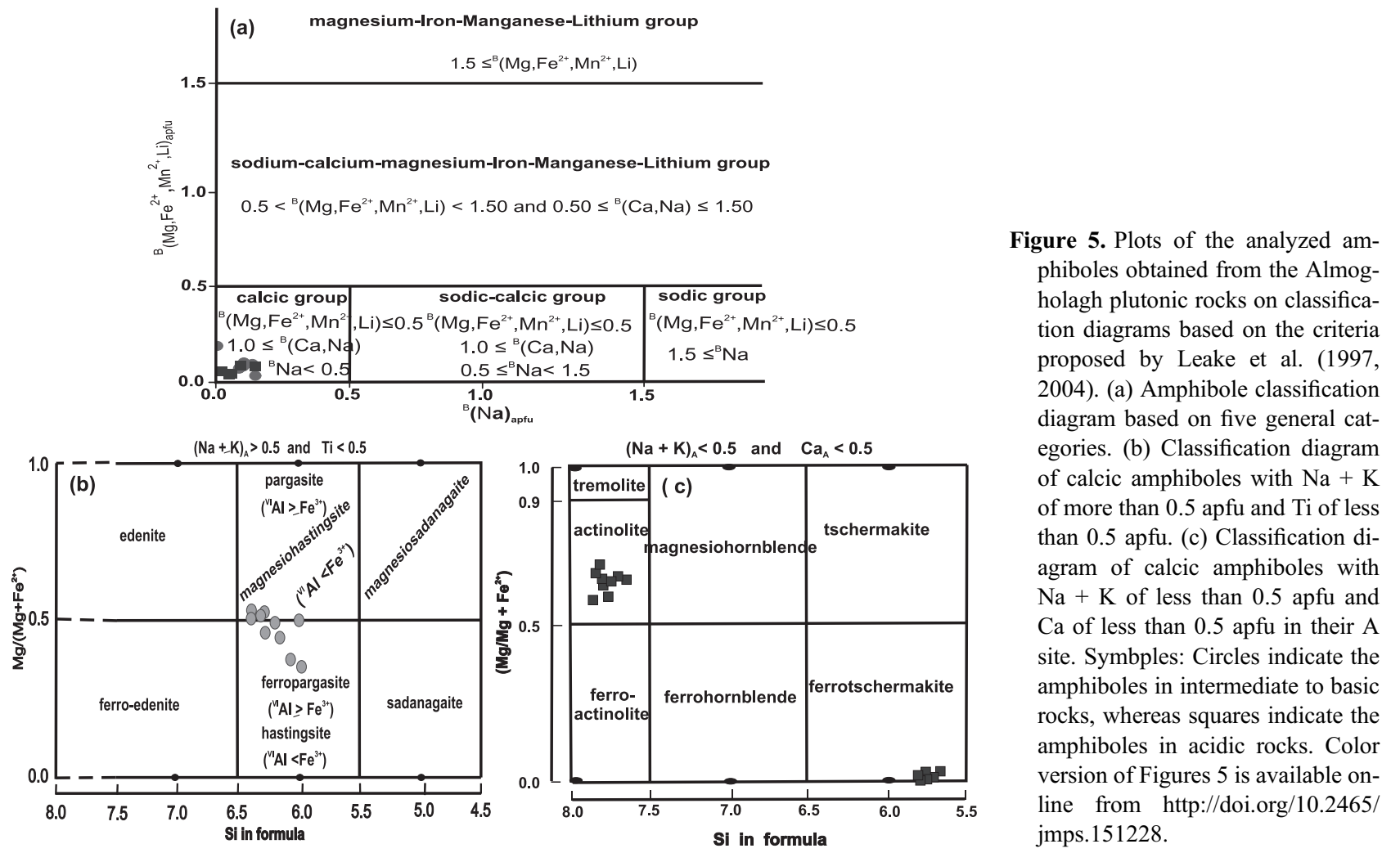


Table 4. Obtained pressures for the analyzed points (in amphiboles)

\begin{tabular}{cccccccc}
\hline Spots & \multicolumn{2}{c}{ Empirical } & Geobarometers & \multicolumn{2}{c}{ Experimental } & Geobarometers & \multicolumn{3}{c}{ Average } \\
\cline { 2 - 7 } Am48-1 & Formula 1 & Formula 2 & Formula 3 & Formula 4 & Empirical & Experimental & Total \\
Am48-2 & 7.1 & 7.6 & 5.8 & 7.4 & 7.3 & 6.6 & 6.9 \\
Am48-3 & 6.3 & 6.6 & 5.1 & 6.6 & 6.5 & 5.9 & 6.1 \\
Am48-4 & 6.9 & 7.4 & 5.7 & 7.3 & 7.2 & 6.5 & 6.8 \\
Am47-1 & 6.7 & 7.1 & 5.4 & 7.0 & 6.9 & 6.2 & 6.5 \\
Am47-2 & 6.1 & 6.5 & 5.0 & 6.8 & 6.3 & 5.7 & 6.0 \\
Am47-3 & 7.2 & 7.7 & 5.9 & 7.5 & 7.5 & 6.7 & 7.1 \\
Am46-1 & 8.0 & 8.6 & 6.5 & 8.2 & 8.3 & 7.4 & 7.8 \\
Am46-2 & 7.5 & 8.8 & 6.7 & 8.5 & 8.5 & 7.6 & 8.1 \\
Am46-3 & 8.1 & 8.1 & 6.0 & 7.8 & 7.8 & 6.9 & 7.3 \\
Average & 7.2 & 8.7 & 6.7 & 8.4 & 8.4 & 7.5 & 8.0 \\
\hline
\end{tabular}

the diagrams showed that the amphiboles in the intermediate and basic rocks belong to either the hastingsite or magnesiohastingsite subgroups (Fig. 5b), which contains less than $6.4(\mathrm{Si})$ and $2(\mathrm{Mg})$ apfu. The amphiboles in the acidic rocks belong either to the ferrotschermakite subgroup, which contains less than $5.8(\mathrm{Si})$ and $0.5(\mathrm{Mg})$ apfu, or to the actinolite subgroup (Fig. 5c), which contains more than $7.6(\mathrm{Si})$ and $2.7(\mathrm{Mg})$ apfu. According to Leake (1978), magmatic amphiboles contain less than 7.3 apfu of Si atoms, and amphiboles containing more than 7.3 apfu of Si atoms are derived via the subsolidus process (Agemar et al., 1999). The amphiboles in the acid rocks from the Almogholagh pluton are not suitable for geothermobarometry because the ferrotschermakites do not coexist with plagioclases (they are probably derived from magma that initially crystallized at a considerable depth), and the actinolites were derived through the subsolidus process. None of the amphiboles in the intermediate to basic rocks were formed through the subsolidus process. Thus, all of them are suitable and selected for geothermobarometry.

\section{Geobarometry of amphiboles}

Using the $\mathrm{Al}^{\text {total }}$ value obtained from the structural formula of amphiboles (Table 3) and the four formulas of empirical and experimental geobarometers (formulas 1, 2,3 , and 4 in the APPENDIXES), we calculated the crystallization pressure of the analyzed points (Table 4). The pressure computed by using the $\mathrm{Al}$ in amphiboles shows the crystallization depth of the analyzed points in the amphiboles and may reflect the depth at which hornblendes crystallize rather than the pressure at which granitoid rocks consolidate, that is, upward movement may continue following amphibole crystallization (Ghent et al., 1991). The average pressure of the analyzed points is $\sim 7$ kbar. Using the conversion factor $1 \mathrm{kbar}=3.7 \mathrm{~km}$ for the continental crust (Tulloch and Challis, 2000) and a calculated error factor of $\pm 0.5 \mathrm{kbar}$ for the pressure, we found that the analyzed points crystallized at a depth of $24-28 \mathrm{~km}$.

\section{Geothermobarometry of amphiboles}

To determine the formation temperature of the amphiboles, we first estimated the $\mathrm{Fe}^{2+}$ and $\mathrm{Fe}^{3+}$ contents in accordance with the method employed by Holland and Blundy (1994) and determined the structural formulas of the amphiboles. The aforementioned method is similar to but different to a certain extent from the methods employed by Spear and Kimball (1984) and Robinson et al. (1982). On the basis of the calculated pressures presented in Table 4, the temperatures were calculated by the BH90, $\mathrm{HB}_{1} 94$ and $\mathrm{HB}_{2} 94$ geothermometers, and results are presented in Table 5. The temperatures that were estimated at the pressure derived from the formula established by Schmidt (1992) were selected as initial temperatures. Subsequently, the values for pressure obtained by using the method of Anderson and Smith (1995) were based from the initial temperatures and then placed for iteration in the spreadsheet program (prepared by Lawford Anderson, University of Southern California). Finally, the temperatures of $\mathrm{HB}_{1} 94, \mathrm{HB}_{2} 94$, and $\mathrm{BH} 90$ were calculated. The values for pressure obtained using the formula proposed by Anderson and Smith (1995) were subsequently calculated on the basis of the $\mathrm{HB}_{2} 94$ temperature. According to Anderson (1996), the temperature of $\mathrm{HB}_{2} 94$ is more appropriate to compute for the pressure than the other temperatures.

\section{Intersection of geobarometers with geothermometers}

Sayari (2011) stated that the temperature fluctuates within a certain range and the calculated value does not display 
Table 5. Geothermobarometric results for the analyzed points (in amphiboles)

\begin{tabular}{|c|c|c|c|c|c|c|c|c|c|c|c|}
\hline Spots & $\begin{array}{l}\mathrm{Am} \\
48-1\end{array}$ & $\begin{array}{c}\mathrm{Am} \\
48-2\end{array}$ & $\begin{array}{c}\mathrm{Am} \\
48-3\end{array}$ & $\begin{array}{c}\mathrm{Am} \\
48-4\end{array}$ & $\begin{array}{l}\mathrm{Am} \\
47-1\end{array}$ & $\begin{array}{l}\mathrm{Am} \\
47-2\end{array}$ & $\begin{array}{l}\mathrm{Am} \\
47-3\end{array}$ & $\begin{array}{c}\mathrm{Am} \\
46-1\end{array}$ & $\begin{array}{c}\mathrm{Am} \\
46-2 \\
\end{array}$ & $\begin{array}{l}\mathrm{Am} \\
46-3\end{array}$ & Average \\
\hline \multicolumn{12}{|c|}{ Based on Hammarstrom and Zen (1986) pressure } \\
\hline$P(\mathrm{kbar})$ & 7.1 & 6.3 & 6.9 & 6.7 & 6.1 & 7.2 & 8.0 & 8.2 & 7.5 & 8.1 & 7.2 \\
\hline$T\left({ }^{\circ} \mathrm{C}\right) \mathrm{HB}_{1} 94$ & 731 & 718 & 701 & 728 & 749 & 751 & 733 & 777 & 822 & 799 & 751 \\
\hline$T\left({ }^{\circ} \mathrm{C}\right) \mathrm{HB}_{2} 94$ & 715 & 682 & 595 & 694 & 739 & 712 & 701 & 702 & 798 & 758 & 710 \\
\hline$T\left({ }^{\circ} \mathrm{C}\right) \mathrm{BH} 90$ & 755 & 736 & 718 & 743 & 763 & 756 & 753 & 769 & 808 & 790 & 759 \\
\hline \multicolumn{12}{|c|}{ Based on Hollister et. al (1987) pressure } \\
\hline$P(\mathrm{kbar})$ & 7.6 & 6.6 & 7.4 & 7.1 & 6.5 & 7.7 & 8.6 & 8.8 & 8.1 & 8.7 & 7.7 \\
\hline$T\left({ }^{\circ} \mathrm{C}\right) \mathrm{HB}_{1} 94$ & 729 & 716 & 699 & 725 & 746 & 749 & 731 & 773 & 817 & 797 & 748 \\
\hline$T\left({ }^{\circ} \mathrm{C}\right) \mathrm{HB}_{2} 94$ & 718 & 684 & 597 & 697 & 741 & 715 & 705 & 705 & 801 & 762 & 712 \\
\hline$T\left({ }^{\circ} \mathrm{C}\right) \mathrm{BH} 90$ & 747 & 730 & 711 & 737 & 757 & 748 & 743 & 759 & 799 & 780 & 751 \\
\hline \multicolumn{12}{|c|}{ Based on Schmidt (1992) pressure } \\
\hline$P(\mathrm{kbar})$ & 7.4 & 6.6 & 7.3 & 7.0 & 6.5 & 7.5 & 8.2 & 8.5 & 7.8 & 8.4 & 7.5 \\
\hline$T\left({ }^{\circ} \mathrm{C}\right) \mathrm{HB}_{1} 94$ & 730 & 716 & 700 & 726 & 747 & 750 & 732 & 776 & 820 & 798 & 749 \\
\hline$T\left({ }^{\circ} \mathrm{C}\right) \mathrm{HB}_{2} 94$ & 717 & 684 & 596 & 696 & 741 & 713 & 703 & 703 & 800 & 760 & 711 \\
\hline$T\left({ }^{\circ} \mathrm{C}\right) \mathrm{BH} 90$ & 750 & 731 & 713 & 738 & 757 & 751 & 748 & 765 & 803 & 786 & 754 \\
\hline \multicolumn{12}{|c|}{ Based on Johnson and Rutherford (1989) pressure } \\
\hline$P$ (kbar) & 5.8 & 5.1 & 5.7 & 5.4 & 5.0 & 5.9 & 6.5 & 6.7 & 6.0 & 6.7 & 5.9 \\
\hline$T\left({ }^{\circ} \mathrm{C}\right) \mathrm{HB}_{1} 94$ & 735 & 723 & 706 & 733 & 755 & 759 & 736 & 787 & 835 & 803 & 757 \\
\hline$T\left({ }^{\circ} \mathrm{C}\right) \mathrm{HB}_{2} 94$ & 707 & 676 & 588 & 688 & 733 & 705 & 692 & 695 & 793 & 749 & 703 \\
\hline$T\left({ }^{\circ} \mathrm{C}\right) \mathrm{BH} 90$ & 775 & 754 & 737 & 762 & 781 & 777 & 775 & 793 & 833 & 814 & 780 \\
\hline \multicolumn{12}{|c|}{ Based on average empirical (Hammarstrom and Zen,1986 + Hollister et. Al, 1987) ) pressure } \\
\hline$P(\mathrm{kbar})$ & 7.3 & 6.5 & 7.2 & 6.9 & 6.3 & 7.5 & 8.3 & 8.5 & 7.8 & 8.4 & 7.5 \\
\hline$T\left({ }^{\circ} \mathrm{C}\right) \mathrm{HB}_{1} 94$ & 730 & 717 & 701 & 727 & 747 & 750 & 732 & 775 & 820 & 798 & 750 \\
\hline$T\left({ }^{\circ} \mathrm{C}\right) \mathrm{HB}_{2} 94$ & 716 & 683 & 596 & 696 & 740 & 713 & 703 & 703 & 800 & 760 & 711 \\
\hline$T\left({ }^{\circ} \mathrm{C}\right) \mathrm{BH} 90$ & 751 & 733 & 714 & 740 & 760 & 752 & 748 & 764 & 803 & 785 & 755 \\
\hline \multicolumn{12}{|c|}{ Based on average experimental (Schmidt,1992 + Johnson and Rutherford, 1989) pressure } \\
\hline$P(\mathrm{kbar})$ & 6.6 & 5.9 & 6.5 & 6.2 & 5.7 & 6.7 & 7.4 & 7.6 & 6.9 & 7.5 & 6.7 \\
\hline$T\left({ }^{\circ} \mathrm{C}\right) \mathrm{HB}_{1} 94$ & 733 & 720 & 703 & 730 & 751 & 754 & 734 & 781 & 827 & 800 & 743 \\
\hline$T\left({ }^{\circ} \mathrm{C}\right) \mathrm{HB}_{2} 94$ & 712 & 680 & 592 & 692 & 737 & 709 & 697 & 699 & 796 & 755 & 707 \\
\hline$T\left({ }^{\circ} \mathrm{C}\right) \mathrm{BH} 90$ & 762 & 742 & 725 & 750 & 769 & 764 & 762 & 779 & 818 & 800 & 767 \\
\hline \multicolumn{12}{|c|}{ Based on total average (average of experimental and empirical pressure) pressure } \\
\hline$P($ Kbar $)$ & 7.0 & 6.1 & 6.8 & 6.5 & 6.0 & 7.1 & 7.8 & 8.1 & 7.3 & 8.0 & 7.1 \\
\hline$T\left({ }^{\circ} \mathrm{C}\right) \mathrm{HB}_{1} 94$ & 731 & 718 & 702 & 728 & 749 & 752 & 733 & 778 & 823 & 799 & 751 \\
\hline$T\left({ }^{\circ} \mathrm{C}\right) \mathrm{HB}_{2} 94$ & 714 & 681 & 594 & 694 & 739 & 711 & 700 & 701 & 798 & 758 & 709 \\
\hline$T\left({ }^{\circ} \mathrm{C}\right) \mathrm{BH} 90$ & 756 & 738 & 720 & 745 & 765 & 758 & 755 & 771 & 811 & 793 & 761 \\
\hline \multicolumn{12}{|c|}{ Based on iteration using Anderson and Smith (1995) pressure at various thermometers } \\
\hline$T\left({ }^{\circ} \mathrm{C}\right) \mathrm{BH} 90$ & 784 & 751 & 727 & 764 & 795 & 789 & 787 & 823 & 918 & 875 & 801 \\
\hline$P$ (kbar) BH90 & 5.2 & 5.3 & 6.4 & 5.3 & 4.1 & 5.1 & 5.8 & 4.8 & 0.7 & 2.9 & 4.5 \\
\hline$T\left({ }^{\circ} \mathrm{C}\right) \mathrm{HB}_{1} 94$ & 733 & 719 & 702 & 731 & 754 & 759 & 735 & 792 & 862 & 806 & 759 \\
\hline$P(\mathrm{kbar}) \mathrm{HB}_{1} 94$ & 6.4 & 5.9 & 6.8 & 6.0 & 5.1 & 5.9 & 7.1 & 5.8 & 3.0 & 5.3 & 5.7 \\
\hline$T\left({ }^{\circ} \mathrm{C}\right) \mathrm{HB}_{2} 94^{*}$ & 713 & 683 & 599 & 695 & 736 & 710 & 700 & 701 & 790 & 750 & 708 \\
\hline$P($ kbar $) \mathrm{HB}_{2} 94$ & 6.8 & 6.5 & 7.9 & 6.7 & 5.5 & 6.9 & 7.8 & 8.0 & 5.3 & 6.9 & 6.8 \\
\hline \multicolumn{12}{|c|}{ Based on finnal Anderson and Smith (1995) pressure (calculated by $T\left({ }^{\circ} \mathrm{C}\right) \mathrm{HB}_{2} 94^{*}$ ) } \\
\hline$P(\mathrm{kbar})$ & 6.8 & 6.5 & 6.6 & 6.7 & 5.5 & 7.0 & 7.8 & 8.0 & 6.0 & 6.9 & 6.8 \\
\hline$T\left({ }^{\circ} \mathrm{C}\right) \mathrm{HB}_{1} 94$ & 732 & 717 & 703 & 727 & 752 & 753 & 733 & 779 & 835 & 802 & 753 \\
\hline$T\left({ }^{\circ} \mathrm{C}\right) \mathrm{HB}_{2} 94$ & 713 & 683 & 593 & 695 & 736 & 711 & 700 & 701 & 793 & 751 & 708 \\
\hline$T\left({ }^{\circ} \mathrm{C}\right) \mathrm{BH} 90$ & 759 & 732 & 723 & 742 & 773 & 760 & 755 & 772 & 833 & 810 & 766 \\
\hline Average of $T$ & 735 & 713 & 672 & 723 & 752 & 742 & 731 & 753 & 817 & 787 & 742 \\
\hline Average of $P$ & 6.7 & 6.1 & 6.9 & 6.4 & 5.7 & 6.8 & 7.6 & 7.6 & 6.0 & 7.8 & 6.7 \\
\hline
\end{tabular}

the desired precision when pressure is calculated using a geobarometer and its value is used in a geothermometer. When the equations are drawn as graphs, the geothermometric curves display low values for the changes in pressure $(d P)$ /changes in temperature $(d T)$ ratio in the $P-T$ diagram. In other words, geothermometers are highly sensitive to temperature variations but are hardly dependent on pressure. By contrast, geobarometers are characterized by high $d P / d T$ values as revealed when they are drawn on a $P-T$ diagram, indicating that they are consid- 
Table 6. Results of the intersections between geobarometers and geothermometers

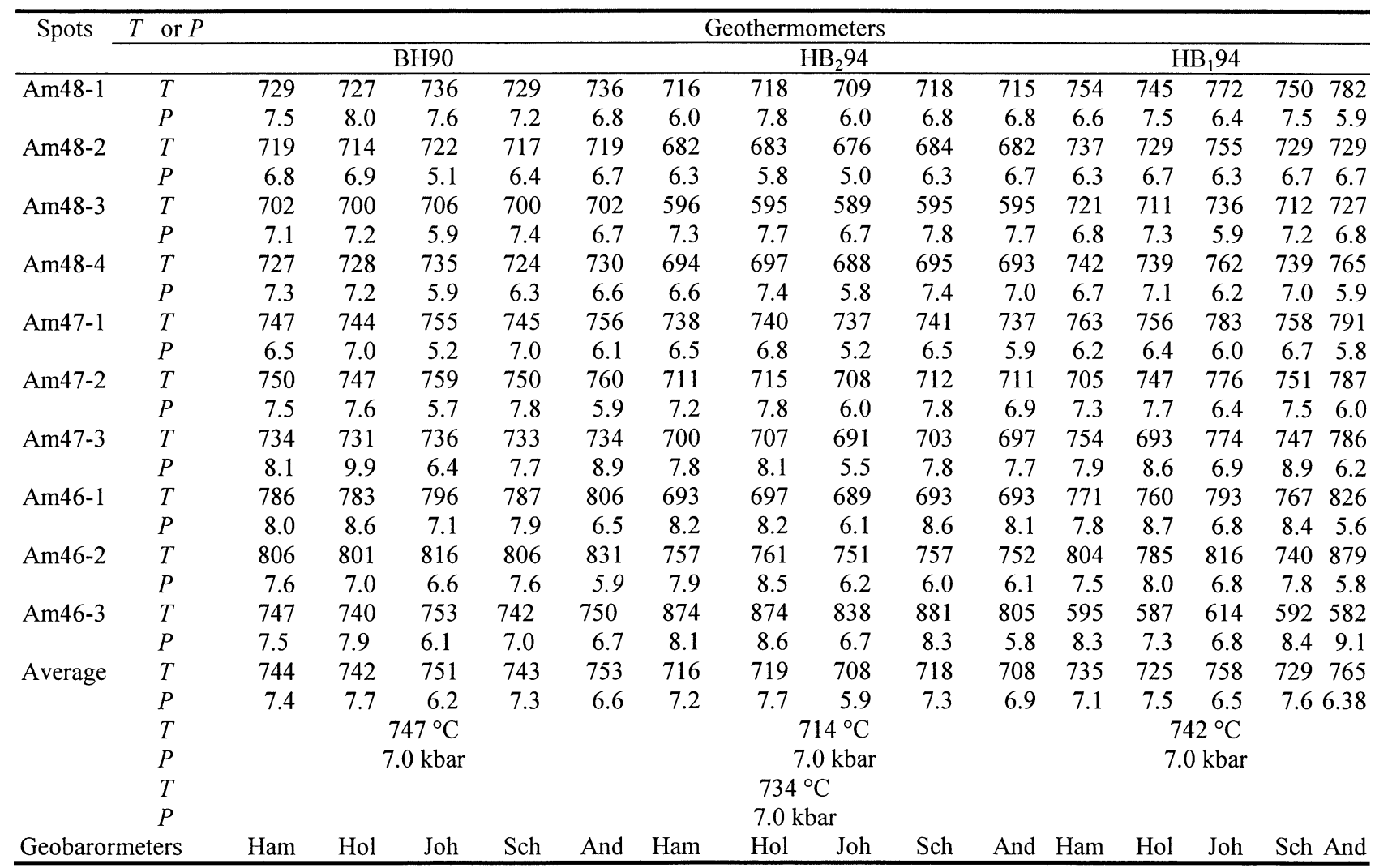

Ham, formula 1; Hol, formula 2; Joh, formula 3; Sch, formula 4; And, formula 5; BH90, fomula 6; $\mathrm{HB}_{1} 94$, formula 7; $\mathrm{HB}_{2} 94$, formula 8 (in the APPENDIXES)

erably more sensitive to pressure variations than to temperature variations. Therefore, these two aforementioned curves intersect at a point that more accurately represents the crystallization temperature and pressure. The three geothermometers (i.e., BH90, $\mathrm{HB}_{1} 94$, and $\mathrm{HB}_{2} 94$ ) in the top row of Table 6 intersected with five geobarometers (i.e., Ham, Hol, Jon, Sch, and And) in the bottom row of Table 6 , and the pressure and temperature from their intersection were determined (Table 6). The average pressure and temperature of the analyzed points in the amphiboles are $7.0 \mathrm{kbar}$ and $734{ }^{\circ} \mathrm{C}$ respectively, which are approximately equal to the average values $(6.7 \mathrm{kbar}$ and $742{ }^{\circ} \mathrm{C}$ ) in Table 5 . In addition, the pressure is equal to the average values $(7.1 \mathrm{kbar})$ in Table 4 . These differences are not significant at a $95 \%$ confidence interval.

A relatively high correlation between temperature and pressure was observed in Table $5\left(R^{2}=0.99\right)$ and Table $6\left(R^{2}=0.8\right)$. The equations showing the relationship between temperature and pressure show that the temperature changes by $\sim 5-10{ }^{\circ} \mathrm{C}$ when the pressure changes by 1 kbar.

\section{Intersection of geothermometers}

As mentioned earlier, geobarometers and geothermometers are sensitive to $d P$ and $d T$, respectively. Therefore, the intersection of a geobarometer with a geothermometer determines the appropriate pressure because both curves consist of a pressure parameter and at least one of which is sensitive to $d P$. However, the use of the intersection to express the temperature may not be suitable because only one of two curves consists of a temperature parameter. By contrast, in the intersection of two geothermometers, both curves consist of a temperature parameter and are sensitive to temperature changes; therefore, the average temperature obtained from the intersection of geothermometers with one another is closer to the real temperature, although the pressure resulting from their intersection is not accurate and is different from the real pressure because none of the curves are sensitive to pressure. The intersection of $\mathrm{BH} 90$ and $\mathrm{HB}_{2} 94$ for point $\mathrm{Am} 47-2$ is shown in Fig. 6 as an example. The three geothermometers, namely, $\mathrm{BH} 90, \mathrm{HB}_{1} 94$, and $\mathrm{HB}_{2} 94$, were intersected with one another for all analyzed points (with the ex- 


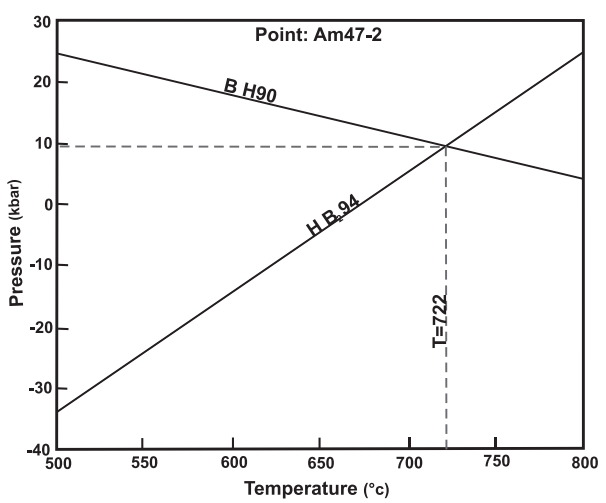

Figure 6. Intersection of $\mathrm{BH} 90$ and $\mathrm{HB}_{2} 94$ for $\mathrm{AM} 47-2$ point. Color version of Figures 6 is available online from http:// doi.org/10.2465/jmps. 151228 .

Table 7. Temperatures at the intersections of two pair geothermometers

\begin{tabular}{ccc}
\hline Spots & $\mathrm{HB}_{1} 94$ with $\mathrm{HB}_{2} 94$ & $\mathrm{HB}_{2} 94$ with BH90 \\
\hline Am48-1 & 720 & 735 \\
$\mathrm{Am} 48-2$ & 701 & 694 \\
$\mathrm{Am} 48-3$ & 654 & 624 \\
$\mathrm{Am} 48-4$ & 711 & 706 \\
$\mathrm{Am} 47-1$ & 744 & 742 \\
$\mathrm{Am} 47-2$ & 730 & 722 \\
Am47-3 & 711 & 713 \\
Am46-1 & 729 & 711 \\
Am46-2 & 728 & 709 \\
Am46-3 & 752 & 782 \\
average & 718 & 714 \\
Toal average & & $716^{\circ} \mathrm{C}$ \\
\hline
\end{tabular}

ception of $\mathrm{HB}_{1} 94$ with $\mathrm{BH} 90$ ), and the results of their intersections are presented in Table 7. The average temperatures obtained from the two intersecting pairs of geothermometers are similar at a $95 \%$ confidence interval. Their average temperature $\left(716^{\circ} \mathrm{C}\right)$ differs from the temperature in Table $5\left(742{ }^{\circ} \mathrm{C}\right)$ by only $\sim 26^{\circ} \mathrm{C}$, which is not significant at a $95 \%$ confidence interval.

The average temperatures of $\mathrm{HB}_{2} 94$ given in Tables 5 and 6 are $709^{\circ} \mathrm{C}$ and $714{ }^{\circ} \mathrm{C}$, respectively, and they are considerably close to the obtained average results of the intersection of geothermometers $\left(716^{\circ} \mathrm{C}\right.$, Table 7). This result demonstrates that this geothermometer $\left(\mathrm{HB}_{2} 94\right)$ can provide a good estimate of the crystallization temperature in the intermediate to basic rocks. A relatively high correlation exists between the temperature and pressure that were obtained by the $\mathrm{HB}_{2} 94$ geothermometer (Table $\left.5, R^{2}=0.999\right)$. The equation between temperature and pressure (Fig. 7) shows that the temperature changed by $\sim 5{ }^{\circ} \mathrm{C}$ against a pressure of $1 \mathrm{kbar}$. However, if the calculated error factor for pressure is assumed to be \pm 0.5

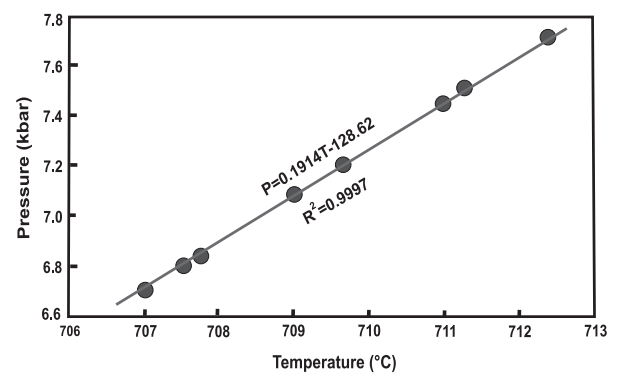

Figure 7. Correlation between the average temperature and the average pressure from the $\mathrm{HB}_{2} 94$ geothermometer in amphiboles (based on Table 5). Color version of Figures 7 is available online from http://doi.org/10.2465/jmps.151228.

kbar, the temperature change is equal to $2.5^{\circ} \mathrm{C}$. Therefore, the average crystallization temperature using the intersection of geothermometers of the analyzed points in the amphiboles is $714-719^{\circ} \mathrm{C}$. According to the different equations of geothermometers (APPENDIXES), we assumed that the crystallization temperature changes than the average temperature is equivalent to $\pm 40{ }^{\circ} \mathrm{C}$. In summary, the amphiboles in intermediate to basic rocks of the area crystallized at a depth of $24-28 \mathrm{~km}$ of the crust and at $674-759^{\circ} \mathrm{C}$.

The aforementioned temperatures were confirmed by zircon saturation thermometry $\left(T_{Z r}\right) \cdot T_{Z r}$ provides a simple and robust means to estimate the temperatures of magma (Watson, 1979). Zircon saturation temperatures calculated from bulk-rock compositions provide the minimum estimates of melt temperature if the magma was undersaturated with $\mathrm{Zr}$ and the maxima if the magma was saturated with $\mathrm{Zr}$ (Miller et al., 2003; Zhao et al., 2012). The temperatures of the melt system were estimated by using $\mathrm{Zr}$ solubility in granitoid melts as proposed by Watson (1979) and Watson and Harrison (1983). Their experiments showed that the partition coefficient $\left(D_{Z r}\right.$ zircon) is a function of the parameter $M=(\mathrm{Na}+\mathrm{K}+2 \mathrm{Ca}) /$ $(\mathrm{Si} \times \mathrm{Al})$ and temperature. If a granitic melt is saturated with $\mathrm{Zr}$, then its temperature may be calculated from the measured major element and $\mathrm{Zr}$ contents. The calculated zircon saturation temperatures for Almogholagh intermediate to basic rocks (I-type) range from $591{ }^{\circ} \mathrm{C}$ to 741 ${ }^{\circ} \mathrm{C}$ with an average of $679^{\circ} \mathrm{C}$, which is significantly lower than that of typical A-type rocks in the area. The presence of a significant amount of amphiboles ( 30-35\%) in intermediate to basic rocks from the Almogholagh pluton of calc-alkaline affinity (I-type magma) shows that the hydrous conditions play an important role in magma formation. The presence of a significant amount of $\mathrm{H}_{2} \mathrm{O}$ in a liquid stabilizes amphiboles during the crystallization of magma and alters its physical properties (low viscosity, low density, and low liquidus/solidus) and phase re- 
lations (Anderson 1980). Nevertheless, on the basis of $T_{Z r}$ results (Watson and Harrison, 1983), Almogholagh intermediate to basic rocks apparently formed from melts at low temperatures, which are probably related to the high $\mathrm{Zr}$ at the source and to the presence of fluid influx (Miller et al., 2003). As stated earlier, the source of all magma forming in the Almogholagh pluton contains high $\mathrm{Zr}$ and probably is affected by a high fluid flux of mantle-derived halogen-rich volatiles.

\section{CONCLUSIONS}

The following conclusions are drawn on the basis of our data:

1. The use of the average pressure obtained from the intersections of geothermometers with geobarometers is suitable to determine the amount of pressure in the magma during the crystallization of the analyzed points, whereas the intersection among geothermometers can be used to determine the crystallization temperature.

2. The average temperature of the $\mathrm{HB}_{2} 94$ geothermometer is approximately equal to the average temperature of the intersection among the geothermometers. $\mathrm{HB}_{2} 94$ is apparently a suitable geothermometer to determine the crystallization temperature in intermediate to basic rocks.

3. The average crystallization temperature and pressure obtained for the analyzed points are $716^{\circ} \mathrm{C}$ and $7 \mathrm{kbar}$, respectively. However, if the calculated error factor for pressure is assumed to be $\pm 0.5 \mathrm{kbar}$, the average crystallization temperature of the analyzed points of the amphiboles ranges from 714 to $719^{\circ} \mathrm{C}$, and the amphiboles in intermediate to basic rocks of the area crystallized at a depth of $24-28 \mathrm{~km}$ at $674-759^{\circ} \mathrm{C}$.

\section{ACKNOWLEDGMENTS}

This work was conducted as a part of the PhD Thesis of M. Amiri. The authors would like to thank Dr. H. Shafei Moghadam for the chemical analysis of rock samples at the ACME Analytical Laboratories in Canada. We are grateful to anonymous reviewers for their critical and constructive comments for the improvement of our manuscript. We are thankful to Dr. Masaaki Owada for his comments and editorial handling.

\section{SUPPLEMENTARY MATERIALS}

Color versions of Figures 1-7 are available online from http://doi.org/10.2465/jmps.151228.

\section{REFERENCES}

Agard, P., Omrani, J., Jolivet, L. and Mouthereau, F. (2005) Convergence history across Zagros (Iran): constraints from collisional and earlier deformation. International Journal of Earth Science, 94, 401-419.

Agemar, T., Worner, G. and Heumann, A. (1999) Stable isotopes and amphibole chemistry on hydrothermally altered granitoids in the North Chilean Precordillera: a limited role for meteoric water? Contributions to Mineralogy and Petrology, 136, 331344.

Ague, J.J. (1997) Thermodynamic calculation of emplacement pressures for batholithic rocks, California: Implications for the aluminum-in-hornblende barometer. Geology, 25, 563566.

Ague, J.J. and Brandon, M.T. (1992) Tilt and northward offset of Cordilleran batholiths resolved using Igneous barometry. Nature, 360, 146-149.

Alavi, M. (1994) Tectonics of Zagros orogenic belt of Iran: new data and interpretation. Tectonophysics, 229, 211-238.

Amiri, M. (2015) Geochemical and petrological characteristics of Almogholagh Batholit (northren Asadabad, Hamedan Province, West of Iran). pp. 254, PhD.Thesis, Lorestan University, Iran (in Persian).

Anderson, J.L. (1996) Status of thermobarometry in granitic batholiths. Earth sciences, 87, 125-138.

Anderson, A.T., Jr. (1980) Significance of hornblende in calc-alkaline andesites and basalts. American Mineralogist, 65, 837851.

Anderson, J.L. and Smith, D.R. (1995) The effect of temperature and oxygen fugacity on Al-in-Hornblende barometry. American Mineralogist, 80, 549-559.

Azizi, H. and Moinevaziri, H. (2009) Review of the tectonic setting of Cretaceous to Quaternary volcanism in northwestern Iran. Journal of Geodynamics, 47, 167-179.

Berberian, F. and Berberian, M. (1981) Tectono-plutonic episodes in Iran. Geological Survey of Iran, 52, 565-592.

Blundy, J.D. and Holland, T.J.B. (1990) Calcic amphibole equilibria and a new amphibole-plagioclase geothermometer. Contributions to Mineralogy and Petrology, 104, 208-224.

Chappell, B.W. and White, A.J.R. (1974) Two contrasting granite types. Pasific Geology, 8, 173-174.

Cox, K.G., Bell, J.D. and Pankhurst, R.J. (1979) The interpretation of igneous rocks. pp. 450, Springer/Chapman and Hall, London.

Debon, F. and Le Fort, P. (1983) A chemical- mineralogical classification of common plutonic rocks and associations. Transactions of the Royal Society of Edinburgh Earth Sciences, 73, 135-149.

De La Roche, H., Leterrier, J., Grandclaude, P. and Marchal, M. (1980) A classification of volcanic and plutonic rocks using $\mathrm{R}_{1} \mathrm{R}_{2}$-diagram and major element analyses-its relationships with current nomenclature. Chemical Geology, 29, 183-210.

Eftekharnejad, J. (1981) Tectonic division of Iran with respect to sedimentary basins. Journal of Iranian Petroleum Society, 82, 19-28.

Ernst, W.G. (2002) Paragenesis and thermobarometry of Ca-amphiboles in the Barcroft granodioritic pluton, central White Mountains, eastern California. American Mineralogist, 87, 478-490.

Ghalamghash, J., Nédélec, A., Bellon, H., Vousoughi Abedini, M. 
and Bouchez, J.L. (2009) The Urumieh plutonic complex (NW Iran): a record of the geodynamic evolution of the Sanandaj-Sirjan zone during Cretaceous times-Part I: Petrogenesis and K/Ar dating. Journal of Asian Earth Sciences, 35, 401-415.

Ghent, E.D., Nicholls, J., Siminy, P.S., Sevigny, H.H. and Stout, M.Z. (1991) Hornblende Geobarometry of the Nelson batholith, Southeastern British Columbia: Tectonic Implications. Canadian Journal of Earth Sciences, 28, 1982-1991.

Golonka, J. (2004) Plate tectonic evolution of the southern margin of Eurasiain the Mesozoic and Cenozoic. Tectonophysics, 381, 235-273.

Hammarstrom, J.M. and Zen, E-a. (1986) Aluminum in hornblende: an empirical igneous geobarometer. American Mineralogist, 71, 1297-1313.

Harris, N.B.W. and Marriner, G.F. (1980) Geochemistry and petrogenesis of a peralkaline granite complex from the Midian Mountains, Saudi Arabia. Lithos, 13, 325-337.

Harris, N.B.W., Pearce, J.A. and Tindle, A.G. (1986) Geochemical characteristics of collision-zone magmatism. Collision Tectonics, Geological Society, Special Publications, 19, 67-81.

Helmy, H.M., Ahmed, A.F., El Mahallawi, M.M. and Ali, S.M. (2004) Pressure, temperature and oxygen fugacity conditions of calc-alkaline granitoids, Eastern Desert of Egypt, and tectonic implication. Journal of African Earth Science, 38, 255268.

Holland, T. and Blundy, J. (1994). Non-ideal interactions in calcicamphiboles and their bearing on amphibole-plagioclase thermometry. Contributions to Mineralogy and Petrology, 116, 433-447.

Hollister, L.S., Grissom, G.e., Peters, E.K., Stowell, H.H. and Sisson, V.R. (1987) Confirmation of the empirical correlation of $\mathrm{AI}$ in hornblende with pressure of solidification of calc-alkaline plutons. American Mineralogist, 72, 231-239.

Irvine, T.N. and Baragar, W.R.A. (1971) A guide to the chemical classification of the common volcanic rocks. Canadian Journal of Earth Scinces, 8, 523-548.

Johnson, M.C. and Rutherford, M.J. (1989) Experimental calibration of the aluminum-in-hornblende geobarometer with application to long valley caldera (California) volcanic rock. Geology, 17, 837-841.

Leake, B.E. (1971) On aluminous and edenitic hornblendes. Mineralogical Magazine, 38, 389-407.

Leake, B.E. (1978) Nomenclature of amphiboles. American Mineralogist, 63, 1023-1052.

Leake, B.E., Woolley, A.R., Arps, C.E.S., Birch, W.D., Gilbert, M.C., Grice, J.D., Hawthorne, F.C., Kato, A., Kisch, H.J., Krivovichev, V.G., Linthout, K., Laird, J., Mandarino, J.A., Maresch, W.V., Nickel, E.H., Rock, N.M.S., Schumacher, J.C., Smith, D.C., Stephenson, N.C.N., Ungaretti, L., Whittaker, E.J.W. and Youzhi, G. (1997) Nomenclature of amphiboles: Report of the Subcommittee on Amphiboles of the International Mineralogical Association, Commission on New Minerals and Mineral Names. American Mineralogist, 82, 1019-1037.

Leake, B.E., Woolley, A.R., Birch, W.D., Burke, E.A.J., Ferraris, G., Grice, J.D., Hawthorne, F.C., Kisch, H.J., Krivovichev, V.G., Schumacher, J.C., Stephenson, N.C.N. and Whittaker, E.J.W. (2004) Nomenclature of amphiboles: Additions and revisions to the International Mineralogical Association's amphibole nomenclature. American Mineralogist, 89, 883-887.

Mazhari, S.A., Bea, F., Amini, S., Ghalamghash, J., Molina, J.F.,
Montero, M.P., Scarrow, J. and Williams, I.S. (2009) The Eocene bimodal Piranshahr massif of the Sanandaj-Sirjan Zone, NW Iran: a marker of the end of the collision in the Zagros orogen. Journal of the Geological Society, 166, 53-69.

McDonough, W.F. and Sun, S.-S. (1995) The composition of the Earth. Chemical Geology, 120, 223-253.

Mengel, F.C. and Rivers, T. (1991) Decompression reactions and $\mathrm{P}-\mathrm{T}$ condition in high-grade rocks, northern Labador: $\mathrm{P}-\mathrm{T}$ paths from individual samples and implications for carly proterozoic tectonic evolution. J. Petrol, 32, 139-167.

Middlemost, E.A.K. (1994) Naming materials in the magma/igneous rock system. Earth Science Reviews, 37, 215-224.

Miller, C.F., McDowell, S.M. and Mapes, R.W. (2003) Hot and cold granites? Implications of zircon saturation temperatures and preservation of inheritance. Geology, 31, 529-532.

Mohajjel, M., Fergusson, C.L. and Sahandi, M.R. (2003) Cretaceous-Tertiary convergence and continental collision, Sanandaj-Sirjan zone eastern Iran. Journal of Asian Earth Sciences, 21, 397-412.

Mohajjel, M. and Izadi Kian, L. (2007) Poly-deformed tectonites in dome structure of the Almogholagh region, West of Hamedan. Geosciences Scientific Journal, 66, 116-113.

Moody, J.B., Meyer, D. and Jenkins, J.E. (1983) Experimental characterization of the greenschist/amphibolite boundary in mafic systems. American Journal of Science, 283, 48-92.

Poli, S. and Schmidt, M.W. (1992) A comment on Calcic amphibole equilibria and a new amphibole-plagioclase thermometer by J.D. Blundy and T.J.B. Holland (1990). Contributions to Mineralogy and Petrology, 111, 273-282.

Robinson, P., Spear, F.S., Schumachumacher, J.C., Laird, J., Klein, C., Evans, B.W. and Doolan, B.L. (1982) Phase relations of metamorphic amphiboles: natural occurrence and theory. Mineralogical Society of America, Reviews in Mineralogy. 9B.1-227.

Sayari, M. (2011) APG: An efficient software program for Amp-Pl thermobarometry based on graphical method. Journal of Sciences, Islamic Republic of Iran, 22, 345-349.

Schmidt, M.W. (1992) Amphibole composition in tonalite as a function of pressure: An experimental calibration of the Alin-hornblende barometer. Contributions to Mineralogy and Petrology, 110, 304-310.

Sengör, A.M.C. (1984) The Cimmeride orogenic system and the tectonics of Eurasia. Geological Society of America, Special Paper, 82, 195.

Sepahi, A.A., Jafari, S.R. and Mani-Kashani, S. (2009) Low pressure migmatites from the Sanandaj-Sirjan metamorphic belt in the Hamedan region (Iran). Geologica Carpathica, 60, 107119.

Shahbazi, H., Siebel, W., Pourmoafee, M., Ghorbani, M., Sepahi, A.A., Shang, C.K. and Vousoughi Abedini, M. (2010) Geochemistry and $\mathrm{U}-\mathrm{Pb}$ zircon geochronology of the Alvand plutonic complex in Sanandaj-Sirjan Zone (Iran): New evidence for Jurassic magmatism. Journal of Asian Earth Sciences, 39, 668-683.

Shahbazi, H., Sieble, W., Ghorbani, M., Pourmoafee, M., Sepahi, A.A., Vousoughi Abedini, M. and Shang, C.K. (2015) The Almogholagh pluton, Sanandj-Sirjan Zone, Iran: geochemistry, U-(Th)-Pb Titanite geochronology and implication for its Tectonic evolution. Neues Jahrbuch für Mineralogie - Abhandlungen, 192, 85-99.

Spear, F.S. and Kimball, K. (1984) RECAMP-A FORTRAN IV program for estimating $\mathrm{Fe}^{3+}$ contents in amphiboles. Comput- 
ers and Geosciences, 10, 317-325.

Stein, E. and Dietl, E. (2001) Hornblende thermobarometry of granitoids from the central Odenwald (Germany) and their implication for the geotectonic development of the Odenwald. Mineralogy and Petrology, 72, 185-207.

Stocklin, J. and Nabavi, M. (1972) Tectonic map of Iran, Scale 1:2500000. Geological Survey of Iran.

Tulloch, A.J. and Challis, G.A. (2000) Emplacement depths of Paleozoic-Mesozoic plutons from western New Zealand estimated by Hornblende-Al Geobarometry. New Zea-land Journal of Geology and Geophysics, 43, 555-567.

Valizadeh, M.V. and Zarian, S. (1976) A petrological study of the Almogholagh, Asadabad and Hamedan plutons. Journal of Science (university of Tehran), 8, 49-59 (in Persian).

Watson, E.B. (1979) Zircon saturation in felsic liquids: experimental data and applications to trace element geochemistry. Contributions to Mineralogy and Petrology, 70, 407-419.
Watson, E.B. and Harrison, T.M. (1983) Zircon saturation revisited: temperature and composition effects in a variety of crustal magma types. Earth and Planetary Science Letters 64, 295304.

Whalen, J.B., Currie, K.L. and Chappell, B.W. (1987) A-type granites: geochemical characteristics, discrimination and petrogenesis. Contributions to Mineralogy and Petrology 95, 407-419.

Zhao, K.D., Jiang, S.Y., Chen, W.F., Chen, P.R. and Ling, H.F. (2012) Zircon U-Pb chronology and elemental and Sr-NdHf isotope geochemistry of two Triassic A-type granites in South China: implication for petrogenesis and Indosinian transtensional tectonism. Lithos, 160-161, 292-306.

Manuscript received December 28, 2015

Manuscript accepted May 9, 2016

Published online October 5, 2016

Manuscript handled by Masaaki Owada

\section{APPENDIXES}

The Geobarometry and geothermometry equations were used for calculating the pressure or temperature and mentioned in the text, as equations 1 to 8 have been presented in the below. In all formulas, the temperature $(T)$ is in centigrade degree $\left({ }^{\circ} \mathrm{C}\right)$ and the pressure $(P)$ is in kilobar (kbar).

1) $P( \pm 3 \mathrm{kbar})=-3.92+5.03 \mathrm{Al}^{\text {total }} \quad$ (Hammarstrom and Zen, 1986)

2) $P( \pm 1 \mathrm{kbar})=-4.76+5.64 \mathrm{Al}^{\text {total }} \quad$ (Hollister et al., 1987)

3) $P( \pm 1 \mathrm{kbar})=-3.46+4.23 \mathrm{Al}^{\text {total }} \quad$ (Johnson and Rutherford, 1989)

4) $P( \pm 0.6 \mathrm{kbar})=-3.01+4.76 \mathrm{Al}^{\text {total }} \quad$ (Schmidt, 1992)

5) $P( \pm 0.6 \mathrm{kbar})=4.76 \mathrm{Al} l^{\text {total }}-3.01-\left[\left(\frac{T_{\left({ }^{\circ} \mathrm{C}\right)}-675}{85}\right) \times\left[0.53 \mathrm{Al} l^{\text {total }}+0.005294\left(T_{\left({ }^{\circ} \mathrm{C}\right)}-675\right)\right]\right]$

(Anderson and Smith, 1995)

6) $T_{B H 90}\left( \pm 40^{\circ} \mathrm{C}\right)=\frac{0.677 P-48.98}{-0.0429-0.0083144 \ln \left[\left(\frac{S i-4}{8-S i}\right) \cdot X_{a b}^{P l}\right]} \quad$ (Blundy and Holland, 1990)

7) $T_{H B_{1} 94}=T_{A}\left( \pm 40^{\circ} C\right)=\frac{-76.95+0.79 P+Y_{a b}+39.4 X_{N a}^{A}+22.4 X_{K}^{A}+(41.5-2.89 P) \cdot X_{A l}^{M_{2}}}{-0.0650-0.0083144 \ln \left(\frac{27 X_{V a c}^{A} X_{S i}^{T_{1}} X_{a b}^{P l}}{276 X_{N a}^{A} X_{A l}^{T_{1}}}\right)}-273.15$

(Holland and Blundy, 1994)

In above formula, for $X_{a b}^{P l}>0.5$, then $Y_{a b}=0.0$ otherwise $Y_{a b}=12.0\left(1-X_{a b}^{p l}\right)^{2}-3.0 \mathrm{KJ}$

8) $T_{H B_{2} 94}=T_{B}\left( \pm 40^{\circ} C\right)=\frac{78.44+Y_{a b-a n}-33.6 X_{N a}^{M_{4}}-(66.8-2.92 P) \cdot X_{A l}^{M_{2}}+78.5 X_{A l}^{T_{1}}+9.4 X_{N a}^{A}}{0.0721-0.0083144 \ln \left(\frac{27 X_{N a}^{M_{4}} \cdot X_{S i}^{T_{1}} \cdot X_{a n}^{P l}}{64 X_{C a}^{M_{4}} \cdot X_{A l}^{T_{1}} \cdot X_{a b}^{P l}}\right)}-273.15$

(Holland and Blundy, 1994)

In above formula, for $X_{a b}^{p l}>0.5$, then $Y_{a b-a n}=3.0 \mathrm{KJ}$ otherwise $Y_{a b-a n}=12.0\left(2 . X_{a b}^{p l}-1\right)+3.0 K J$ 